\title{
Prediction of Recrystallization Times in Electroplated Copper Thin Films
}

Mikhail Treger ${ }^{1}$, Christian Witt ${ }^{2}$, Cyril Cabral Jr. ${ }^{3}$, Conal Murray ${ }^{3}$, Jean Jordan-Sweet ${ }^{3}$, Robert Rosenberg ${ }^{4}$, Eric Eisenbraun ${ }^{4}$, I.C. Noyan ${ }^{1, *}$

${ }^{1}$ Department of Applied Physics and Applied Mathematics, Columbia University, New York 10027, USA

${ }^{2}$ GlobalFoundries Inc., Malta, New York 12020, USA

${ }^{3}$ IBM T.J. Watson Research Center, Yorktown Heights, New York 10598, USA

${ }^{4}$ The College of Nanoscale Science and Engineering at SUNY Polytechnic Institute, Albany, New York 12203, USA

\begin{abstract}
:
Room temperature recrystallization responses of blanket electroplated Cu thin films deposited under various conditions were monitored in real-time using synchrotron $\mathrm{x}$-ray diffraction. Nominal control of electroplating parameters such as plating current, bath chemistry, and plating time was found to be insufficient to ensure repeatability of the $50 \%$ recrystallization time, $\tau_{50}$, from sample to sample even though the thickness variations between samples were insignificant. Real-time x-ray analysis of samples from numerous electroplating baths showed that, for a given seed deposition process, a reliable estimation of $\tau_{50}$ at room temperature can be obtained from the ratio of the integrated intensities of the 111 and $200 \mathrm{Cu}$ reflections, $\overline{\mathrm{I}}_{111} / \overline{\mathrm{I}}_{200}$, of the electroplated film at time zero (immediately after plating). Among the plating parameters investigated seed-layer texture most influenced this ratio and, hence, the subsequent room temperature recrystallization behavior of the plated film.
\end{abstract}

\section{1-Introduction}

Freshly electroplated copper thin films can undergo large microstructural changes at room temperature. Despite several decades of research there is no consensus on the energetics and kinetics associated with these changes [1]. Part of the problem is due to the lack of repeatability between electroplated samples. Typically, ensuring repeatability in electroplating 
is approached through imposing tight controls on plating current, time, and chemistry [2-12]. However films plated under nominally identical conditions can show different recrystallization responses; samples obtained from different areas of a single wafer can also show differences in recrystallization times [4, 7]. These effects are usually ascribed to thickness variations. To obtain a better metric of sample repeatability we used real-time synchrotron $\mathrm{x}$-ray diffraction $[3,13]$ to study microstructural changes from electroplated films deposited with a variety of frequently employed, phenomenological plating parameters. These included plating time and film thickness[4, 13], seed layer deposition methodology[5, 6], seed layer and plated film texture $[7,8]$, diffusion barrier chemistry[9, 10], and impurity concentration[11, 12].

Our results, summarized in Figure 1, showed that $50 \%$ recrystallization times $\left(\tau_{50}\right)$ were strongly correlated with the ratio of the integrated intensities of the 111 and 200 Cu reflections, $R_{T x}=\overline{\mathrm{I}}_{111} / \overline{\mathrm{I}}_{200}$, of the electroplated film at time zero, $\left(\tau=\tau_{0}\right)$. This observation agrees with the findings by Ueno et al. [7] who reported results from a much smaller sample set (three samples). In addition we observe the presence of a critical initial texture ratio, $R_{T x}^{*}$, which separates two regions where the dependency of $\tau_{50}$ on $R_{T x}$ is opposite. For $R_{T x}>R_{T x}^{*}, \tau_{50}$ increases with increasing $R_{T x}$. For $R_{T x}<R_{T x}^{*}$, the opposite trend is observed. Over each range $R_{T x}$ can be used to predict $\tau_{50}$, independent of the film thickness and other process parameters. Thus, this ratio can be utilized as a reliable metric for the a priori estimation of the recrystallization times of electroplated blanket Cu thin films, unlike prior studies $[11,14,15]$, which were based on impurity or plating chemistry dependent indicators.

\section{II-Analysis Technique}

X-ray diffraction experiments record the intensity of elastically scattered $\mathrm{x}$-ray photons by a crystalline sample as a function of scattering angle, 20. For a polycrystalline thin film the measured intensity is the sum of the diffracted intensities from all illuminated grains satisfying Bragg's law:

$$
\begin{aligned}
& \mathrm{I}_{h k l}(\Delta 2 \theta)=\sum_{j} \mathrm{~N}_{\mathrm{j}}^{2}\left|\mathrm{~F}_{\mathrm{hkl}}\right|^{2} \operatorname{Sinc}^{2}(\mathrm{t} \vartheta) \\
& \vartheta=\frac{\pi}{\lambda} \cos \theta_{\mathrm{B}}(\Delta 2 \theta) ; \Delta 2 \theta=2 \theta-2 \theta_{\mathrm{B}}
\end{aligned}
$$


In Eqn. 1, $\mathrm{N}_{\mathrm{j}}$ is the number of unit cells in a diffracting grain, and the summation is over all diffracting grains for the particular reflection at Bragg angle $\theta_{B} ; \mathrm{F}_{\mathrm{hkl}}$ is the structure factor for this reflection $h k l, t$ is the film thickness, and $\lambda$ is the wavelength of the monochromatic $x$-rays [3]. Since maximum peak intensity is difficult to measure experimentally, the integrated peak intensity, $\overline{\mathrm{I}}_{\mathrm{hkl}}=\int \mathrm{I}(2 \theta) \mathrm{d}(2 \theta)$, which is less susceptible to experimental and statistical errors[16] and is directly proportional to the diffracting grain volume for the $h k /$ reflection, is generally used. The integrated intensity, $\overline{\mathrm{I}}_{\mathrm{hkl}}$, is proportional to the total volume of crystalline material which contributes finite intensity to the measured Bragg peak. The full-width-at halfmaximum intensity (FWHM) of the Bragg peak, $\beta_{\mathrm{hkl}}$, can be used to determine the equivalent (effective) grain size, $\langle D\rangle_{h k l}$ along the film thickness direction through the Scherer equation:

$\langle D\rangle_{h k l}=\frac{C \lambda}{\beta_{h k l} \cos \theta_{B}}$

Here the carats indicate the diffraction average taken over all diffracting grains. For films with columnar microstructures, $\langle D\rangle_{h k l}=t$. It is seen that monitoring $\overline{\mathrm{I}}_{\mathrm{hkl}}$ and $\beta_{\mathrm{hkl}}$ as a functions of time can yield quantitative data about variations in the volume and thickness of the transforming regions associated with the particular reflection [13]. We note that using the FWHM to monitor the transformation requires more effort since the broadening contributions from all sources -such as instrumental, coherent domain size and lattice parameter dispersion terms must be separated[1].

In our current study the diffraction data analysis followed the procedures described in our previous studies $[3,13]$, where a model that coupled time-resolved x-ray diffraction measurements to the traditional Johnson-Mehl-Avrami-Kolmogorov (JMAK) kinetics analysis [17] was reported. In the classic JMAK analysis, Eqn. 3, $f$ is the recrystallized volume fraction within the region of interest, $\tau_{0}$ is the incubation time, $\tau$ is the elapsed time, $m$ is a constant related to the dimensionality of the recrystallization, and $K$ is a parameter encompassing nucleation and growth rates.

$\mathrm{f}(\tau)=1-\mathrm{e}^{\left(-\mathrm{K}\left(\tau-\tau_{0}\right)^{\mathrm{m}}\right)}$ 
In addition to $K$ and $m$, the parameters $\tau_{50}$ and $\tau_{\infty}$ the time required to achieve $50 \%$ and $100 \%$ of the transformation, respectively, are also used to characterize the transformation. In the literature the $\tau_{50}$ parameter is the preferred metric for comparing recrystallization times of different samples [11].

For x-ray diffraction measurements the recrystallized volume fraction within the region of interest, $f$, is expressed in terms of the normalized change in the integrated intensity, $\overline{\mathrm{I}}_{\mathrm{hkl}}$, for a particular reflection:

$f_{\overline{\mathrm{I}}_{\mathrm{hkl}}}(\tau)=\frac{\overline{\mathrm{I}}_{\mathrm{hkl}}(\tau)-\overline{\mathrm{I}}_{\mathrm{hkl}}\left(\tau_{0}\right)}{\overline{\mathrm{I}}_{\mathrm{hkl}}\left(\tau_{\infty}\right)-\overline{\mathrm{I}}_{\mathrm{hkl}}\left(\tau_{0}\right)}$

Data obtained from Eqn. 4 refers only to transformation within the grains diffracting into the hkl reflection employed in the measurement. For a symmetric, radial scan all of these (diffracting) grains have one member of their $\langle\mathrm{hkl}>$ vectors parallel to the surface normal of the thin film; thus the ensemble of the diffracting grains form a (discontinuous) volume with virtual fiber texture, with the fiber axis being along the $\langle\mathrm{hkl}\rangle$ direction [18]. For the purposes of this paper we will refer to these (virtual) fiber texture sets as "texture components". Consequently the transformation kinetics parameters, $K, m, \tau_{0}, \tau_{50}$, and $\tau_{\infty}$, obtained from a diffractionbased JMAK analysis are texture-specific, and can be different for different reflections.

\section{III- Experimental Details}

\section{III-1 Sample Manufacture}

A standard sample metallurgy was selected based on its industrial relevance. A schematic of the standard film stack is shown in Figure 2. The dual-layer diffusion barrier of $10 \mathrm{~nm}$ cubic F $\alpha$-Ta on $5 \mathrm{~nm}$ TaN (amorphous) of this standard template was physical-vapor-deposited (PVD) in an Endura sputtering system on a $0.7 \mathrm{~mm}$ thick, $200 \mathrm{~mm}$ diameter, silicon (001) wafer. Subsequently, a $50 \mathrm{~nm}$ Cu seed layer was deposited by sputter PVD at $10 \mathrm{mTorr}$ argon partial pressure (1.33Pa) without breaking vacuum.

The wafer was then cleaved into coupons, approximately $8 \times 8 \mathrm{~mm}^{2}$, on which Cu thin films were electroplated manually in a beaker. The electroplating solution consisted of $40 \mathrm{~g} / \mathrm{l} \mathrm{Cu}, 10 \mathrm{~g} / \mathrm{I}$ 
$\mathrm{H}_{2} \mathrm{SO}_{4}, 50 \mathrm{ppm} \mathrm{Cl}$, with $6 \mathrm{ml} / /$ commercially available Viaform ${ }^{\circledR}$ accelerator and $2 \mathrm{ml} / /$ Viaform ${ }^{\circledR}$ suppressor. It was vigorously stirred during plating. An undoped, high purity $\mathrm{Cu}$ sheet was used as the anode. The electroplated region was confined to a circular area in the center of each coupon by masking the surface with dicing tape in which a $4 \mathrm{~mm}$ diameter hole had been punched.

To enhance Cu nucleation, each sample was initially exposed to a low plating current density of $5.0 \mathrm{~mA} / \mathrm{cm}^{2}$ for approximately 10 seconds. Subsequently, a higher current density of 50 $\mathrm{mA} / \mathrm{cm}^{2}$ was applied. The application time of the higher current density determined film thickness: if applied for 80 seconds, an electroplated Cu layer with nominal thickness of $1.0 \mu \mathrm{m}$ was obtained. To prevent transformation during transportation, the plated samples were immediately submersed under liquid nitrogen in a Dewar flask. Accurate film thickness values were obtained by focused ion beam milling and scanning electron microscopy (FIB/SEM) after the $\mathrm{x}$-ray measurements. Some thickness values were also validated by profilometry.

\section{III-2 X-ray Measurements}

Diffraction measurements were performed at beamline X20A of the National Synchrotron Light Source at Brookhaven National Laboratory using a Huber 5020 four-circle diffractometer. A double-crystal Ge (111) monochromator was used to obtain either a 0.25 or $1 \mathrm{~mm}^{2}$ incident $\mathrm{x}$ ray beam spot on the sample with an energy of $9.200 \mathrm{keV}(\lambda=1.348 \AA$ ). A Ge (111) analyzer crystal, placed in front of the avalanche photodiode detector, was used for high angular resolution. The incident direct beam, measured with these optics, exhibited a Lorentzian profile with a FWHM of $0.009 \pm .001$ degrees. For all samples, symmetric radial scans of the 111, 200, 220 and 311 Cu reflections were obtained; typical scan times were 5-10 minutes per reflection. The saturation of the diffracted intensities from the minor texture components $(200,220$, and 311 reflections) always occurred prior to or coincided with the saturation of the diffracted intensity from dominant 111 grain volume. On this basis the monitoring of the 111 reflection intensity, $\overline{\mathrm{I}}_{111}$, was sufficient to determine the overall recrystallization time of each film. Therefore, all tabulated references to recrystallization time, $\tau_{50}$, are drawn from the JMAK 
fittings of the 111 reflections [19]. Further details of the experimental procedure are reported in our previous articles $[3,13]$.

\section{IV- Experimental Results}

\section{IV-1 Correlation between Film Recrystallization Time and Initial Film Texture:}

In industrial practice it has been commonly assumed that controlling plating chemistry, plating current, and plating time would be sufficient to yield repeatable thickness and recrystallization behavior in the resulting electroplated $\mathrm{Cu}$ film. There are many studies that show that such control does yield electroplated films of comparable thickness [20,21]. However, we were not able to find published statistical studies that validated the uniformity of recrystallization response. Our first set of experiments was designed to test this assumption. Seven nominally identical samples were prepared under nominally identical plating chemistry, plating current, and plating time. The coupons for these samples were all cleaved from a single wafer on which a $50 \mathrm{~nm}$ PVD Cu seed had been deposited at $10 \mathrm{mTorr}$ argon partial pressure (1.33 Pa) over a dual-layer diffusion barrier of $10 \mathrm{~nm}$ Ta on $5 \mathrm{~nm}$ TaN. The coupons were plated sequentially in the same beaker containing the same plating bath chemistry for $80 \pm 1$ seconds with the same plating current sequence $\left(5.0 \mathrm{~mA} / \mathrm{cm}^{2}\right.$ for 10 seconds, then $50 \mathrm{~mA} / \mathrm{cm}^{2}$ for 70 seconds). The samples were then transported to NSLS in liquid nitrogen, and the diffraction responses of the recrystallizing films were measured in real time. Changes in $\overline{\mathrm{I}}_{111}$ versus time for these seven samples are shown in Figure 3. It is seen that the transformation responses for these nominally identical samples are not identical. Transformation parameters obtained from these curves through the JMAK analysis, listed in the first three columns of Table 1, show that the recrystallization times, $\tau_{50}$, computed from the 111 integrated intensities of these "identical" samples vary by as much as $\pm 20 \%$.

Comparing the intensities of the 200, 220 and 311 reflections with that of the 111 reflection it was seen that all seven electroplated films possessed strong 111 fiber textures; the 200 reflections yielded the next highest diffracted intensity for all samples. Consequently, the ratio of the integrated intensities of the 111 and 200 reflections at time zero, $R_{T x}$, was used to 
quantify the strength of the initial 111 texture of the electroplated film on a given coupon. These values are shown in the fifth column of Table 1: all films exhibit very strong initial 111 texture, with $R_{T X}=\overline{\mathrm{I}}_{111} / \overline{\mathrm{I}}_{200}$ ratios in excess of $80: 1$. In untextured copper films the $\overline{\mathrm{I}}_{111} /$ $\overline{\mathrm{I}}_{200}$ ratio would be approximately 2:1 (JCPDS \#040836).

Comparison of the second and fifth columns of Table 1 shows that lower initial film texture ratios, $R_{T x}$, correlate with faster sample recrystallization times, $\tau_{50}$ (Fig. 1 ; black squares). The spread of $R_{T x}$ values is quite similar to that of the $\tau_{50}$ values; in both cases the standard deviation is approximately $23 \%$ of the mean value. We also note that in the JMAK analysis, Eqn. 3 , the constant related to the dimensionality of the recrystallization, $m$, shows the smallest standard deviation, approx. $\pm 6 \%$, while the $K$ parameter, encompassing nucleation and growth rates, shows variability in excess of $100 \%$.

\section{IV-2 Effect of Cu Seed Layer Texture:}

Since the variability in the $\tau_{50}$ values listed in Table 1 cannot be ascribed to the plating parameters, other possible causes were investigated. In a previous study where full wafers were used, In the past literature several groups, including Ueno and co-workers, ascribed such differences to variability in thickness [7-10, 12]. In our work, no variation of plating power across the small plating area is expected, and profilometry measurements and FIB cross sections coupled with SEM analysis confirmed that uniform thickness was achieved for all coupons. Since it is also well known that the initial texture of the electroplated Cu films can be influenced by the texture of the Cu seed layers [7-10,12] through the "templating" or "texture inheritance" mechanisms [12], texture variation within the seed layer was identified as a second possibility. To test this hypothesis, 111 peaks of the Cu seed layers of all seven samples were measured from the masked regions of each coupon (Figure 4). These protected seed layer regions were stable at room temperature and did not show any evidence of transformation during our experiments. On the other hand, PVD seed and electroplated Cu film layers could not be distinguished in cross-sectional FIB/SEM micrographs after the recrystallization of the overlying film, indicating consumption of the seed layer. Figure 4 shows that both maximum 
and integrated intensities of the 111 peaks of the seed layers, as well as their FWHM values, $\beta_{\mathrm{hkl}}$, showed significant variability between nominally identical coupons.

The (maximum) difference in integrated intensities of the weakest and strongest 111 reflections in Figure 4, measured from the seed layers of samples PVD-10mT-1 and PVD-10mT-5 respectively, is $36 \%$ of the overall mean integrated intensity from all seven coupons, with one standard deviation corresponding to $12 \%$ of this mean value. These variations can be caused by variations in the film thickness, film texture, or both of film thickness and texture. In general, while a slight variation of PVD seed thickness from the center to the edge of a wafer is observed, $\pm 20 \%$ variability in film thickness is not expected in coupons cut from adjacent portions at center of the wafer. SEM/FIB imaging (Figure 5) also indicated a uniform Cu seed.

Based on these observations, we ascribed the variations in the 111 peaks to the changes in the texture of the underlying $\mathrm{Cu}$ seed and concluded that the repeatability of the texture of the electroplated film was strongly influenced by the repeatability of the texture of the seed layer. This conclusion was then tested by adapting the approach of Clevenger, et al. [22], who modulated the stress and microstructure of Ta films by varying the argon partial pressure in the chamber, to Cu seed layer deposition.

In this series of tests $50 \mathrm{~nm}$ thick Cu seed layers were grown using physically vapor deposited (PVD) in an Endura sputtering system on two $0.7 \mathrm{~mm}$ thick, $200 \mathrm{~mm}$ diameter, silicon (001) wafers at 2 mTorr and 25 mTorr of argon partial pressure (0.267 Pa, 3.33Pa), respectively. These wafers were otherwise identical to the standard PVD wafer described in section A. The $8 \times 8 \mathrm{~mm}^{2}$ coupons cleaved from these two wafers were then electroplated with $\mathrm{Cu}$ using the bath chemistry and electroplating procedures described previously. Consequently, the only difference between these samples and the ones used to populate Table 1 is the argon partial pressure used during seed deposition. Diffraction spectra from the seed layers, and the corresponding transformation curves of the electroplated Cu films are shown in Figures $6 a-b$, and $6 \mathrm{c}-\mathrm{d}$ for the $2 \mathrm{mTorr}$ and $25 \mathrm{mTorr}$ seed wafers, respectively. 
Comparison of Figures 6-a and 6-b shows that the 111 reflections obtained from various coupons of the wafer with the PVD Cu seed film deposited at the higher Ar partial pressure exhibited much better uniformity. The Cu seed layer deposited at the lower Ar partial pressure had a lower average 111 intensity, with higher coupon-to-coupon variability. The mean integrated intensity/standard deviation computed from the five samples shown in Figure 6-b is 3.64 \pm 0.4 . The corresponding value for Figure 6 -a are $2.8 \pm 1.0$. The variability of the transformation curves for the electroplated Cu films plated on these two wafers correlated with the variability of the corresponding $\overline{\mathrm{I}}_{111}$ values of the seed layers (Figures 6-c, $\mathrm{d}$ and Table 2). The variability of the average texture ratios at time zero, $R_{T x}$, also correlates with the variability of the corresponding $\overline{\mathrm{I}}_{111}$ values of the seed layers (Figure 1, blue circles). Consequently, our conclusion in section $A$, linking the variability in room-temperature recrystallization response to the variability in seed texture is justified.

Table 2 also shows that the Cu films electroplated on the PVD seed layers deposited at lower Ar partial pressure , PVD-2mT, were more weakly 111 textured than their high pressure, PVD$25 \mathrm{mT}$, counterparts. This corresponded to significantly faster average recrystallization times.

\section{IV-3 Influence of Plated Film Thickness on the Initial Film Texture Ratio}

In the literature there are many studies that show an inverse correlation between the thickness of an electroplated film and its room temperature transformation time, $\tau_{50}[20,21]$. As we noted earlier, the $50 \mathrm{~nm}$ thick Cu seed layer films were stable at room temperature and did not show any evidence of transformation during our experiments. These seed layers were very highly 111 textured; no other reflections yielded finite intensities. Based on our observations in sections $A$ and $B$, we postulated that increasing the thickness of the electroplated film probably resulted in a decrease in the texture ratio at time zero, $R_{T x}$, which in turn, resulted in a faster transformation rate. To test this hypothesis we used a set of samples which were electroplated on coupons cut from the same wafer as the films of Table 1, and therefore possessing nominally identical seed and barrier layer structures. The electroplating process used nominally identical plating current and plating bath chemistry as well. The range of plated film thicknesses was 
accomplished by varying the plating time between 20 to 80 seconds. Sample designations and transformation parameters are shown in Table 3. The numerical part of the sample names (PVD-\#) indicates the duration of sample exposure to the $50 \mathrm{~mA} / \mathrm{cm}^{2}$ current density (in seconds) during electroplating. The film thickness values listed in column 2 were measured by FIB/SEM analysis after the room temperature recrystallization measurements were completed. Comparison of the second and third columns of Table 3 shows that the initial texture ratio of these films, $R_{T x}=\overline{\mathrm{I}}_{111} /\left.\overline{\mathrm{I}}_{200}\right|_{\tau=\tau_{0}}$, monotonically decreases with increasing film thickness. The $R_{T x}$ parameter decreases nearly 7-fold as thickness increases 2.5 times, from $0.4 \mu \mathrm{m}$ to $1.0 \mu \mathrm{m}$. Thus, the strength of the initial 111 texture of an electroplated Cu film changes dramatically with film thickness for films with PVD seed layers. Comparison of the third and fourth columns of Table 3 show that the $50 \%$ transformation time, $\tau_{50}$, decreases monotonically with decreasing texture ratio at time zero, $R_{T x}$. These data fit the trend, established by samples described in Sections $\mathrm{A}$ and $\mathrm{B}$, of the dependency of $\tau_{50}$ on $R_{T x}$; they are depicted by red triangles in Figure 1. The thinner electroplated films of this study have the highest texture ratios, and the slowest transformation times among the samples we studied. We note that the thinnest electroplated films of this set, 0.4 and $0.55 \mu \mathrm{m}$ in thickness, respectively, did not transform during the time available to us at the synchrotron, even though some increase in the 111 intensity was observed [13].

The Cu film electroplated on sample PVD-80 of Table 3 should be nominally identical to the samples described in Table 1. All of these films were plated on coupons from the same wafer, with the same chemistry and voltage/current regimen; in all eight cases the final electroplated films were nominally $1 \mu \mathrm{m}$ thick. There was, however, a six month gap between the two sets of experiments; a new bath, with identical composition, had to be mixed for the second set. We see that the $R_{T x}$ value of sample PVD-80 is approximately $2 x$ that of the average $R_{T x}$ value listed in Table 1. Consequently, even minor composition variations in nominally identical plating baths can result in major changes in the time-zero texture ratio of electroplated films and their subsequent recrystallization behavior. 
At this point comparing the transformation kinetics parameters, $K, m, \tau_{50}$ (Eqn. 3,4$)$ listed in Tables 1, 2 and 3 is also useful. In Tables 1 and 2, the magnitudes of the deviations in the $K$ parameter are similar to their average values. Consequently, the $K$ parameter cannot be used to extract useful information about the transformation for these cases. The average value of the " $m$ " parameter from the PVD-10mT samples (Table 1 ) is similar to the average " $m$ " of the PVD$2 \mathrm{mT}$ samples (Table 2): $3.3 \pm 0.4$ and $3.2 \pm 0.2$, respectively, but not of the PVD- $25 \mathrm{mT}$ samples, $\mathrm{m}$ $=1.6 \pm 0.3$. Both the $2 \mathrm{mT}$ and $10 \mathrm{mT}$ samples exhibit large variations in $\tau_{50}$ whereas better uniformity in $\tau_{50}$ was observed in the $25 \mathrm{mT}$ samples. Thus, it is possible that $m \approx 3$ (typically corresponding to 3-D growth for growth in a fully nucleated material) may be representative of greater microstructural variability than in samples where $m<2$ (typically corresponding to 2-D growth). However, the parameters $K, m, \tau_{50}$ for the thickness study samples (Table 3 ) show a different trend. For this set the seed $\mathrm{Cu}$ layer was also deposited at $10 \mathrm{mT}$ argon pressure. The deviation in the $K$ values, between 10 to $40 \%$ of the mean value, is significantly smaller than the deviations listed in the previous tables. The $m$ value for the PVD-80 sample, (electroplated Cu film thickness $\approx 1 \mu \mathrm{m}$ ) is 1.8 , indicating 2 -D growth, which is very different from the average $\mathrm{m}$ parameter for Table 1, where all samples had nominally $1 \mu \mathrm{m}$ thick electroplated Cu layers. As noted before, the samples described in Table 3 were plated at a different time, but with a nominally identical bath chemistry and electrodeposition parameters. Consequently, it appears that the JMAK kinetics parameters $K, m$, are not correlate-able across plating baths in our study. While we also observe large variations in the texture ratio between baths, the correlation between $\tau_{50}$ and $R_{T x}$ is obeyed (Figure 1 ). Consequently, only these parameters will be used in the remainder of this study.

\section{IV-4 Controlling Recrystallization of Electroplated Cu Films through Seed Texture Manipulation}

The utilization of the atomic layer deposition (ALD) process for depositing a thinner conformal Cu seed layer has been the subject of much recent research $[5,23]$. This technique is based on a self-limiting surface reaction wherein suitable precursors are pulsed over the substrate surface to form a single atomic monolayer. This is followed by a purge of the ALD chamber followed 
with a subsequent pulse, which forms another identical monolayer on the previous one. The ALD technique allows very precise control of the seed layer thickness but the deposition speed is quite slow, limiting the maximum Cu seed layer thickness to below $20 \mathrm{~nm}$ for the process we used. Our ALD Cu seeds were deposited employing AbaCus (Air Liquide, Inc.) and plasmaactivated hydrogen as reactants in a room temperature process. The use of room temperature processing avoided the significant copper agglomeration which is typical of higher temperature deposition processes [23]. The ALD deposition procedure included a vacuum break between the barrier layer and seed deposition steps.

We prepared two types of wafers which incorporated an ALD seed layer within the sample stack (Table 4). The first wafer, termed the ALD sample ( $1^{\text {st }}$ row, Table 4 ) contained only a single $20 \mathrm{~nm}$ thick ALD seed layer, upon which the blanket Cu film was directly electroplated.

Synchrotron x-ray measurements from this bare ALD seed yielded only an extremely broad and extremely weak, barely discernible 111 reflection (Figure 7-a, bottom black trace). The 200, 220 , and 311 reflections could not be resolved. In addition, the grains within the ALD Cu layer could not be resolved in FIB/SEM cross-sectional photographs. Using the Scherer Equation (Eqn. 2) the average Cu grain size within the ALD layer was estimated to be less than $2 \mathrm{~nm}$.

The initial texture ratio of the Cu film, $R_{T x}$, electroplated on the ALD seed layer was much weaker than the values for the films electroplated on PVD seed layers summarized in Tables 1 and 2. The $\tau_{50}$ transformation time for the Cu film electroplated on ALD Cu seed, 2.8 hours, was also much faster than any other film we studied. The third entry of Table 4 lists the transformation characteristics of a Cu film of comparable thickness, plated on our standard 50 nm PVD Cu seed with the same batch as the ALD coupons using identical plating parameters. Comparison of the $R_{T x}$ and $\tau_{50}$ values for the ALD and PVD samples in Table 4 clearly shows the importance of seed layer texture in modulating the transformation speed. This is also seen in Figure 7-b, where the variation of integrated intensity with time plots of this batch of samples are shown.

To further study the effect of seed layer texture templating on transformation kinetics, Cu was electroplated on coupons cleaved from the dual-seed wafer, in which a nominally $20 \mathrm{~nm}$ ALD 
Cu seed layer was deposited on $30 \mathrm{~nm}$ PVD Cu, sputtered at 10 mTorr argon partial pressure (1.33Pa). We expected a more 111 textured ALD seed layer surface through templating from the highly textured PVD Cu layer. The cross-sectional image of this structure is shown in Figure 8. Both layers are discernible, indicating that they have different microstructures. The ALD Cu grains are not resolved. The 111 reflection for this composite seed layer is shown in the middle (red) trace of Figure 7-a. This profile contains the depth-averaged contributions from both seed layers and shows that, on the average, the seed 111 intensity is much stronger than that of the bare ALD layer, but $1 / 3^{\text {rd }}$ weaker than the $50 \mathrm{~nm}$ PVD seed. Figure 7-a also shows that the 111 peak position of the composite Cu layer is shifted to lower $2 \theta$, compared to that of the $50 \mathrm{~nm}$ PVD Cu seed, indicating that the plane spacing of the composite layer in the direction of the film surface normal is larger, indicating a possible change in the plating residual stress acting on the film

Even though we could not resolve the specific texture of the top (ALD) surface of the composite seed layer independently, some templating must have occurred: The $R_{T x}$ value for the electroplated $\mathrm{Cu}$ film was $3 x$ that of the corresponding film plated on the bare ALD seed layer (Table 4, middle row), with a correspondingly longer transformation time (Figure 7-b). The $\tau_{50}$ vs. $R_{T x}$ data for all three films listed in Table 4 also follow the general trends established in Figure 1.

\section{IV-5 Film Thickness Effect in Transformation of Cu layers Plated on ALD Seed:}

Comparison of Tables 1 and 4 shows that the nominally 1 micrometer thick Cu films electroplated on non-textured ALD seed layers transformed much faster than similar films plated on highly 111 textured PVD Cu seed layers. To check if the same effect permitted the transformation of thinner electroplated layers, $\mathrm{Cu}$ films with thicknesses similar to those listed in Table 3 were electroplated on coupons cleaved from the wafer with $20 \mathrm{~nm}$ ALD Cu seed layer. The results from this study are listed in Table 5, where the sample naming convention is identical to that used in Table 3: ALD-\# indicates that the Cu film was electroplated on ALD Cu seed and the \# indicates the duration (seconds) of high plating current application. 
The third column of Table 5 shows that the time-zero texture ratios, $R_{T x}$, for all ALD-\# samples are two to three orders of magnitude lower than the corresponding values for the PVD films listed in Table 3. All of the samples listed in Table 5 transformed. The $0.33 \mu \mathrm{m}$ thick electroplated Cu film (sample ALD-20) was 50\% transformed in 48 hours. In contrast, the PVD$20 \mathrm{~nm}$ film listed in Table 3 did not transform at all in $150 \mathrm{hr}$. The actual thickness of the electroplated film for sample ALD-40 (Table 5) is slightly lower than that of sample PVD-60. However, the $\tau_{50}$ for sample ALD-40 is an order of magnitude faster. Thus, we conclude that the critical film thickness for room temperature recrystallization of $\mathrm{Cu}$ is strongly dependent on the time-zero texture ratio, $R_{T x}$, of the electroplated film.

Table 5 also shows that for Cu films with very low initial texture ratios, $\tau_{50}$ decreases for a modest increase in $R_{T X}$ in this range (Figure 1, the green inverted triangles, the green dashed line) which is opposite of the trend established by all of our previous samples.

\section{IV-6 Studies with Other Modulators of Texture}

Table 6 shows three further studies where the initial texture ratio was changed by modifying the seed layer. The seed stack for the first wafer consisted of $20 \mathrm{~nm}$ of Co evaporated on $30 \mathrm{~nm}$ of PVD Cu seed. The final Cu layer was directly electroplated on the Co layer. The seed stack for the second wafer consisted of $20 \mathrm{~nm}$ of Cu evaporated on $30 \mathrm{~nm}$ of PVD Cu seed. In the third wafer a single, $10 \mathrm{~nm}$ thick, $\beta$-Ta layer was used as the diffusion barrier, upon which the standard $50 \mathrm{~nm}$ thick Cu PVD seed layer was deposited. The Cu films electroplated on these wafers were significantly thicker. For all three samples the initial texture ratios, $R_{T x}$, were greater than the critical texture ratio, $R_{T x}^{*} \sim 7 . R_{T x}$ vs. $\tau_{50}$ data from all three wafers conformed to the general relationship on the right side of $R_{T x}^{*}$ in Figure 1.

\section{IV-7 Effect of Laboratory vs. Industrial Plating}

The samples listed in Tables 1-6 made use of small Si coupons, where the Cu was electroplated manually in a beaker on a bench top. To see if the results of this manual process were industrially relevant, full 8" Si (001) wafers, processed through the standard PVD Cu seed deposition stage, were sent to a vendor for electroplating; an industrial grade (undisclosed 
chemistry) Cu electroplating process was used. The results of this study are summarized in Table 7. Here the number in the sample name (I-PVD-\#) corresponds to the target film thickness in nanometers. FIB measurements showed that actual films were approximately $40 \%$ thicker than the nominal values requested, but that these were uniform across the wafer. X-ray analysis revealed that these films were strongly 111 textured as well. This initial texture decreased 10x as film thickness increased from $0.55 \mu \mathrm{m}$ to $1.15 \mu \mathrm{m}$, which is similar to our observations in the hand-plated PVD samples (Table 3). This texture drop corresponded to a $6 x$ decrease in recrystallization time. $R_{T x}$ vs. $\tau_{50}$ data from all four wafers conformed to the general relationship on the right side of $R_{T x}^{*}$ in Figure 1.

Table 7 shows that correlations established between film thickness, film texture, and recrystallization time using laboratory processes are also applicable to industrial processes.

Finally we note that in our studies we did not explicitly strive to monitor and control impurity concentrations, such as oxygen, in the plating baths or deposition chambers used to manufacture our samples. As described above, to ensure the broadest coverage of plating parameters possible, we did not limit sample manufacture to one single run, a single process, nor to one single facility. The effect of initial texture on the transformation response from all of our specimens is very consistent, indicating that contamination, to a first approximation, is not a primary driver of initial texture, or $\tau_{50}$, as long as nominal care, standard in industrial processing, is maintained during sample manufacture.

\section{Summary and Conclusions}

The data tabulated in Tables 1-7 show that the strength of the 111 texture component of an electroplated $\mathrm{Cu}$ film at time zero is critical in the development of any subsequent room temperature microstructural transformation. Any process which modifies texture of the seed layer, or the texture of the electroplated film at time zero, will change the recrystallization response. A convenient metric to define the texture strength is the ratio of the integrated intensities from 111 and 200 reflections at time zero, $R_{T X}=\overline{\mathrm{I}}_{111} /\left.\overline{\mathrm{I}}_{200}\right|_{\tau=\tau_{0}}$. For a Cu film with randomly oriented grains the $R_{T x}$ parameter should be equal to 2.0. Higher values indicate 
stronger 111 texture, with more grains having a $<111>$ direction parallel to the surface normal of the film than expected within a random orientation distribution.

The data plotted in Figure 1 show that the x-ray JMAK 50\% transformation time, $\tau_{50}$, of the 111 texture component can exhibit two distinct responses based on the value of $R_{T x}$ : For electroplated Cu films with $R_{T x}$ greater than 7 or so, $\tau_{50}$ increases exponentially with increasing $R_{T x}$. For films with $R_{T x}<7$ the opposite trend is observed. Thus, we define a critical initial texture ratio, $R_{T x}^{*} \cong 7$, which separates the two regions where the variation of $\tau_{50}$ with $R_{T x}$ is opposite.

The majority of samples tested in this study had $R_{T x}$ values greater than 7 (due to textured seed layers) and transformed relatively slowly at room temperature. The thinnest films plated on textured seed layers had the highest $R_{T x}$ values $\left(R_{T x}>1400\right)$, and these did not transform at all. We observed that the most effective means of manipulating $R_{T x}$ was through controlling the texture of the Cu seed layer. Cu films plated on highly textured seed layers were also highly textured, most probably through an efficient templating mechanism [12]. $R_{T x}$ values for these electroplated $\mathrm{Cu}$ layers decreased with increasing film thickness, with an attendant increase in transformation speed. For plated films with $R_{T x}>7$, processing steps which did not change $R_{T x}$ did not influence $\tau_{50}$ values. For example, plastic flow induced in the $\mathrm{Cu}$ film immediately after plating by quenching identically plated coupons in 1-liquid nitrogen, 2-dry ice and 3- icewater did not significantly change the $R_{T x}$ values, and no change in the JMAK $50 \%$ transformation time, $\tau_{50}$, was observed [1].

Cu films electroplated on untextured nano-grained Cu seed layers exhibited $R_{T x}$ values between 4 and 6 . All of these samples, including a $330 \mathrm{~nm}$ thick plated film, completely recrystallized at room temperature at much faster rates than those with $R_{T x}>7$ (Fig. 1, Table 5). In contrast, the 400 and $550 \mathrm{~nm}$ thick films plated on textured seed layers (Table 3) did not transform: the former exhibited no recrystallization, and the latter had barely gotten past incubation after $180 \mathrm{hr}$ at room temperature. For a film of given thickness, this observation indicates that the transformation can be controlled by varying $R_{T x}$, for example by using seed layers with the desired texture. We also conclude that changing the texture changes the 
energetics of recrystallization; surface and strain energy minimization terms which depend on texture are just as important as grain boundary energy minimization considered in reference 7.

The critical texture ratio also delineates two regions where the variation of $R_{T x}$ with film thickness displays opposite behaviors (Figure 9-a). This may be ascribed to the templating process which yields an initially untextured plated Cu layer on the seed, which then recrystallizes rapidly during plating to minimize grain boundary and surface energy terms in the presence of a residual stress field. This forms mildly textured plated films by the end of the plating process [24]. In contrast, the first few Cu layers forming on textured seed are already highly 111 textured. As the film grows thicker, minimization of strain energy causes the formation of some 001 type grains, causing the (thicker) films to have (relatively) lower $R_{T x}$ values [24]. Since, for both types of films, the $50 \%$ recrystallization time, $\tau_{50}$, shows a similar dependency on electroplated film thickness (Figure 9-b), the texture data is critical in identifying the mechanisms responsible for room temperature recrystallization. We are working on this problem and will report it in a further manuscript.

In contrast to the very definite relationship between the texture ratio $R_{T x}$ and the $50 \%$ transformation time, $\tau_{50}$ shown in Figure 1 , we could not correlate the JMAK kinetics parameters $K, m$ to verifiable microstructural changes when the plating bath or other deposition parameters were changed. While these parameters might be useful in analyzing transformation within one sample batch, their utility in a general study appears to be limited.

In summary, our work demonstrates that tightly controlling process parameters, such as the bath chemistry, electrodeposition current, voltage and application times, to control the total thickness of an electroplated $\mathrm{Cu}$ film is insufficient to control its room temperature recrystallization response. Local variations in the texture of the seed layer can cause just as large variations in the transformation times as thickness variations. Ensuring uniform texture of the seed layer will yield uniform room temperature recrystallization response for blanket $\mathrm{Cu}$ films. In addition, electroplating on a seed layer with very low texture enables the recrystallization of very thin films which would otherwise not recrystallize, and significantly speeds up the recrystallization of thicker films. The texture ratio is a simple parameter to 
measure on a manufacturing line [25] and can be a good parameter to monitor for process control purposes.

\section{Acknowledgements}

This work was funded by the Semiconductor Research Corporation and supported by their partners at IBM Research Yorktown, Global Foundries, and SUNY Albany. Use of the National Synchrotron Light Source, Brookhaven National Laboratory, was supported by the U.S. Department of Energy, Office of Science, Office of Basic Energy Sciences, under Contract No. DE-AC02-98CH10886.

\section{Bibliography}


[1] M. Treger, Characterization of Room Temperature Recrystallization Kinetics in Electroplated Copper Thin Films, PhD Thesis, Columbia University, New York, 2015.

[2] K.M. Takahashi, M.E. Gross, Transport phenomena that control electroplated copper filling of submicron vias and trenches, J. Electrochem. Soc. 146 (1999) 4499-4503.

[3] M. Treger, C. Witt, C. Cabral, C. Murray, J. Jordan-Sweet, R. Rosenberg, E. Eisenbraun, I.C. Noyan, Characterization of room temperature recrystallization kinetics in electroplated copper thin films with concurrent $\mathrm{x}$-ray diffraction and electrical resistivity measurements, Journal of Applied Physics 113 (2013).

[4] N.J. Park, D.P. Field, M.M. Nowell, R. Besser, Effect of film thickness on the evolution of annealing texture in sputtered copper films, Journal of Electronic Materials 34 (2005) 1500-1508.

[5] A.U. Mane, S.A. Shivashankar, Atomic layer chemical vapour deposition of copper, Materials Science in Semiconductor Processing 7, (2004) 343-347.

[6] J. Mao, E. Eisenbraun, V. Omarjee, A. Korolev, C. Lansalot, C. Dussarrat, in: J. Flake, M. Koyangi, G.S. Mathad, H.S. Rathore, T. Ritzdorf, O. Leonte, P. Ramm, F. Roozeboom (Eds.), Ultra-low Temperature Deposition of Copper Seed Layers by PEALD, ECS Transactions, 33, (2010) 125-135.

[7] K. Ueno, T. Ritzdorf, S. Grace, Seed layer dependence of room-temperature recrystallization in electroplated copper films, Journal of Applied Physics 86 (1999) 4930-4935.

[8] A.S. Budiman, P.R. Besser, C.S. Hau-Riege, A. Marathe, Y.C. Joo, N. Tamura, J.R. Patel, W.D. Nix, Electromigration-Induced Plasticity: Texture Correlation and Implications for Reliability Assessment, Journal of Electronic Materials 38 (2009) 379-391.

[9] M. Stangl, A. Fletcher, J. Acker, H. Wendrock, K. Wetzig, Influence of Ta-based diffusion barriers on the microstructure of copper thin films, Journal of Electronic Materials 36, (2007) 1625-1629.

[10] D. Edelstein, C. Uzoh, C. Cabral, P. DeHaven, P. Buchwalter, A. Simon, E. Cooney, S. Malhotra, D. Klaus, H. Rathore, B. Agarwala, D. Nguyen, A high performance liner for copper Damascene interconnects, Proceedings of the Interconnect Tech. Conf., Burlingame, Ca. IEEE, (2001), 9-11.

[11] S.H. Brongersma, E. Kerr, I. Vervoort, A. Saerens, K. Maex, Grain growth, stress, and impurities in electroplated copper, Journal of Materials Research 17 (2002) 582-589.

[12] M. Stangl, M. Liptak, A. Fletcher, J. Acker, J. Thomas, H. Wendrock, S. Oswald, K. Wetzig, Influence of initial microstructure and impurities on Cu room-temperature recrystallization (self-annealing), Microelectronic Engineering 85 (2008) 534-541.

[13] A. Ying, C. Witt, J. Jordan-Sweet, R. Rosenberg, I.C. Noyan, A quantitative analysis of room temperature recrystallization kinetics in electroplated copper films using high resolution x-ray diffraction, Journal of Applied Physics 109014907 (2011).

[14] S.P. Hau-Riege, C.V. Thompson, In situ transmission electron microscope studies of the kinetics of abnormal grain growth in electroplated copper films, Appl. Phys. Lett. 76 (2000) 309-310

[15] M. Stangl, J. Acker, V. Dittel, W. Gruner, V. Hoffmann, K. Wetzig, Characterization of electroplated copper self-annealing with investigations focused on incorporated impurities, Microelectronic Engineering 82 (2005) 189-195. 
[16] B.E. Warren, X-Ray Diffraction, Addison-Wesley, London, UK, 1969.

[17] M. Avrami, Granulation, Phase Change, and Microstructure - Kinetics of Phase Change. III, J. Chem. Phys. 9 (1941) 177-184.

[18] H.J. Bunge, Texture analysis in Materials Science: Mathematical Methods, Butterworths, 1982.

[19] For $9.200 \mathrm{keV} x$-rays the beam will penetrate throughout the electroplated film, and the variation of the integrated intensity of a given reflection with time will be proportional to the total diffracting volume for that reflection within the total film thickness. We note that the use of the JMAK model implicitly assumes: (1) the growth rate of the recrystallizing grains is constant in all directions and independent of the extent of transformation, and (2) nucleation is continuous, random, and homogeneous throughout the untransformed portions of the film.

[20] M. Stangl, M. Militzer, Modeling self-annealing kinetics in electroplated Cu thin films, Journal of Applied Physics 103, 113521 (2008).

[21] D. Walther, M.E. Gross, K. Evans-Lutterodt, W.L. Brown, M. Oh, S. Merchant, P. Naresh, Room Temperature Recrystallization of Electroplated Copper Thin Films: Methods and Mechanisms, MRS Proceedings 612 D10.1.1 (2000).

[22] L.A. Clevenger, A. Mutscheller, J.M.E. Harper, C. Cabral, K. Barmak, The Relationship Between Deposition Conditions, The Beta To Alpha Phase-Transformation, And StressRelaxation In Tantalum Thin-Films, Journal of Applied Physics 72 (1992) 4918-4924.

[23] J.J. Mao, E. Eisenbraun, V. Omarjee, C. Lansalot, C. Dussarrat, in: O. Ueda, M. Fukuda, S.J. Pearton, E.L. Piner, P. Montangero (Eds.), Low Temperature Copper Deposition by PE-ALD, MRS Proceedings, 1195, 1195-B12-05, (2010).

[24] C.E. Murray, R. Rosenberg, C. Witt, M. Treger, I.C. Noyan, Evolution of strain energy during recrystallization of plated Cu films, Journal of Applied Physics 113, 203515 (2013).

[25] D.S. Kurtz, K.J. Kozaczek, P.R. Moran, Apparatus and method for texture analysis on semiconductor wafers, US Patent 6301330 B1, 2001. 


\section{LIST OF FIGURE CAPTIONS}

Figure 1: Initial $\overline{\mathbf{I}}_{\mathbf{1 1 1}} / \overline{\mathbf{I}}_{\mathbf{2 0 0}}$ ratio for all Cu films used in this study presented as a function of recrystallization time, and grouped by plating batch.

Figure 2: Cross-section schematic of the standard thin-film stack used in this study.

Figure 3: Temporal variation of the integrated intensities of the 111 reflections, $\overline{\mathbf{I}}_{\mathbf{1 1 1}}$, for a series of nominally identical plated films from the same wafer. These electroplated films showed a greater than $20 \%$ spread in recrystallization time, $\boldsymbol{\tau}_{\mathbf{5 0}}$. The specimen numbers do not represent the order of plating.

Figure 4: 111 Bragg peak profiles of the Cu seed layers for coupons PVD-10mT-1 through PVD10mT-7 (Table 1).

Figure 5: FIB/SEM cross-section of the PVD seed layer-diffusion barrier ( $\mathrm{H} 2)$ stack from the wafer used in the repeatability study with samples of Table 1. The average thickness of the seed layer $(\mathrm{H} 1-\mathrm{H} 2)$ is $51 \pm 4 \mathrm{~nm}$.

Figure 6: 111 Bragg peak profiles measured from PVD Cu seed layers deposited under 2 mTorr (0.267 Pa) (a) and $25 \mathrm{mTorr}(3.33 \mathrm{~Pa}$ ) (b) argon partial pressures, respectively. In both cases the Cu seed layers exhibited strong 111 texture. Variation of the 111 integrated intensity, $\overline{\mathbf{I}}_{\mathbf{1 1 1}}$, with time for Cu films electroplated on the PVD Cu seed layers deposited at 2 mTorr (c) and 25 mTorr of argon partial pressure (d). Transformation parameters obtained from JMAK analysis of these curves are listed in Table 2.

Figure 7-a: 111 Bragg peak profiles measured from copper seed layers deposited by the ALD, PVD, and dual-layer ALD/PVD processes (Table 4). (b) Variation of the 111 integrated intensity, $\overline{\mathbf{I}}_{\mathbf{1 1 1}}$, with time for Cu films electroplated on ALD, PVD, and dual-layer seeds.

Figure 8: FIB/SEM of the cross-section of the dual layer seed (Table 4) where nominally $20 \mathrm{~nm}$ of ALD Cu was deposited on $30 \mathrm{~nm}$ of PVD Cu seed. The ALD Cu grains are not resolved.

Figure 9: Variation of the texture ratio at time zero $\left(R_{T X}\right)(\mathbf{a})$ and $50 \%$ recrystallization time (b) with the thickness of Cu films electroplated on PVD and ALD seed layers. In both figures the dashed lines describe least-squares exponential functions fitted to the data. The first $\tau_{50}$ point (red square) in Figure 9-b for the electroplated Cu films plated on PVD seed is an extrapolated value obtained from the fitted function to the first three data points. 


\section{LIST OF TABLE CAPTIONS}

Table 1: JMAK recrystallization parameters, $\boldsymbol{\tau}_{\mathbf{5 0}}, K, m$, and film texture ratio at time zero, $\boldsymbol{R}_{\boldsymbol{T} \boldsymbol{x}}$, seven nominally identical specimens. The recrystallization parameters were computed from the data shown in Figure 3. The specimen numbers do not represent the order of plating.

Table 2: JMAK transformation parameters and zero-time texture ratios $\left(\boldsymbol{R}_{T x}\right)$ of Cu films electroplated on $50 \mathrm{~nm}$ PVD Cu seed layers deposited under low ( 2 mTorr; $0.267 \mathrm{~Pa}$ ) and high (25 mTorr; 3.33Pa) Argon partial pressures.

Table 3: JMAK recrystallization parameters, $\boldsymbol{\tau}_{\mathbf{5 0}}, K, m$, and film texture ratio at time zero, $\boldsymbol{R}_{\boldsymbol{T} x}$, as a function of plated film thickness. All samples utilized here were cleaved from a wafer with a $50 \mathrm{~nm}$ PVD seed layer deposition at $10 \mathrm{mTorr}$ (1.33Pa) Ar partial pressure, and were electroplated under nominally identical conditions for gradually longer times ( 20 to 80 seconds).

Table 4: Transformation parameters of Cu films electroplated on seed layers incorporating an atomic layer deposited (ALD) Cu layer. For both ALD and PVD-ALD films, the seed layer on which the final Cu layer was electroplated was nominally $20 \mathrm{~nm}$ thick ALD Cu. The diffusion barrier layers for all three sets of samples consisted of $10 \mathrm{~nm} \alpha$-Ta on $5 \mathrm{~nm}$ TaN films as shown in Figure 2.

Table 5: Dependence of the $50 \%$ recrystallization time, $\tau_{50}$, and the time-zero texture ratio, $\boldsymbol{R}_{\boldsymbol{T} \boldsymbol{x}}$ on electroplated film thickness when an ALD Cu layer is used as seed.

Table 6: JMAK Recrystallization parameters for Cu films electroplated on evaporated cobalt and copper capped seed layers (first two rows). For these samples the standard diffusion barrier, 10 $\mathrm{nm} \alpha$-Ta on $5 \mathrm{~nm}$ TaN, was used. The seed layer of the sample described in the last row, $50 \mathrm{~nm}$ thick PVD Cu, was deposited on a single $10 \mathrm{~nm}$ thick layer of $\beta$-Ta.

Table 7: Dependence of the $50 \%$ recrystallization time, $\tau_{50}$, and the time-zero texture ratio, $\boldsymbol{R}_{\boldsymbol{T} x}$ on electroplated film thickness for films electroplated on $50 \mathrm{~nm}$ PVD seed layers by a (proprietary) industrial full wafer plating process. 


\section{Tables}

\begin{tabular}{|c|c|c|c|c|}
\hline Coupon ID & $\tau_{50}$ (hours) & $\boldsymbol{K}\left(\mathbf{x ~ 1 0}^{-4}\right)$ & $\boldsymbol{m}$ & $\boldsymbol{R}_{\boldsymbol{T x}}$ \\
\hline PVD-10mT-1 & $21 \pm 2$ & $0.54 \pm 0.38$ & $3.09 \pm 0.23$ & $145 \pm 11$ \\
\hline PVD-10mT-2 & $21 \pm 2$ & $1.1 \pm 0.73$ & $2.86 \pm 0.21$ & $139 \pm 10$ \\
\hline PVD-10mT-3 & $17 \pm 2$ & $0.67 \pm 0.86$ & $3.24 \pm 0.44$ & $107 \pm 7$ \\
\hline PVD-10mT-4 & $15 \pm 2$ & $0.93 \pm 0.5$ & $3.28 \pm 0.20$ & $99 \pm 5$ \\
\hline PVD-10mT-5 & $15 \pm 2$ & $0.69 \pm .76$ & $3.44 \pm 0.41$ & $95 \pm 6$ \\
\hline PVD-10mT-6 & $14 \pm 2$ & $0.97 \pm 0.84$ & $3.38 \pm 0.32$ & $95 \pm 6$ \\
\hline PVD-10mT-7 & $12 \pm 2$ & $4.1 \pm 2.6$ & $3.03 \pm 0.26$ & $77 \pm 5$ \\
\hline Average & $17 \pm 4$ & $1.28 \pm 1.25$ & $3.2 \pm 0.2$ & $109 \pm 25$ \\
\hline
\end{tabular}

Table 1

\begin{tabular}{|c|c|c|c|c|c|}
\hline Sample ID & $\begin{array}{c}\text { Argon PP } \\
\text { (mTorr) }\end{array}$ & $\tau_{50}$ (hours) & $\boldsymbol{R}_{\boldsymbol{T} x}$ & $\boldsymbol{K}\left(\mathbf{x} 10^{-4}\right)$ & $\boldsymbol{m}$ \\
\hline PVD-2mT & 2 & $7.6 \pm 2.0$ & $27 \pm 14$ & $14 \pm 9$ & $3.3 \pm 0.4$ \\
\hline PVD-25mT & 25 & $8.7 \pm 1.2$ & $44 \pm 9$ & $236 \pm 101$ & $1.6 \pm 0.3$ \\
\hline
\end{tabular}

Table 2

\begin{tabular}{|c|c|c|c|c|c|}
\hline Sample ID & $\begin{array}{c}\text { Film Thickness } \\
(\mu \mathrm{m})\end{array}$ & $\boldsymbol{R}_{\boldsymbol{T} \boldsymbol{x}}$ & $\tau_{\mathbf{5 0}}(\mathbf{h r})$ & $\boldsymbol{K}\left(\mathbf{x ~ 1 0 ^ { - 4 } )}\right.$ & $\boldsymbol{m}$ \\
\hline PVD-20 & $0.4 \pm 0.06$ & $1736 \pm 376$ & -- & -- & -- \\
\hline PVD-30 & $0.55 \pm 0.07$ & $1476 \pm 302$ & -- & -- & -- \\
\hline PVD-40 & $0.7 \pm 0.01$ & $854 \pm 87$ & $150 \pm 10$ & $2.9 \pm 0.3$ & $1.56 \pm .02$ \\
\hline PVD-60 & $0.8 \pm 0.03$ & $534 \pm 77$ & $59 \pm 6$ & $3.2 \pm 0.4$ & $1.89 \pm .03$ \\
\hline PVD-80 & $1.0 \pm 0.06$ & $267 \pm 32$ & $40 \pm 10$ & $10 \pm 0.4$ & $1.80 \pm .01$ \\
\hline
\end{tabular}

Table 3 


\begin{tabular}{|c|c|c|c|c|c|}
\hline Sample ID & $\begin{array}{c}\text { Seed Layer } \\
\text { Stack }\end{array}$ & $\tau_{50}$ (hours) & $\boldsymbol{R}_{\boldsymbol{T} x}$ & $\begin{array}{c}\text { Seed } \\
\overline{\mathbf{I}}_{\mathbf{1 1}} \\
(\mathrm{ct} / \mathrm{kmon} \\
\text { ) }\end{array}$ & $\begin{array}{c}\text { Electroplated } \\
\text { Film Thickness } \\
(\mu \mathrm{m})\end{array}$ \\
\hline ALD & $20 \mathrm{~nm}$ ALD & $2.8 \pm 0.3$ & $6 \pm 1$ & $\begin{array}{c}3 \mathrm{E}-4 \pm 7 \mathrm{E}- \\
5\end{array}$ & $1.35 \pm 0.12$ \\
\hline PVD-ALD & $\begin{array}{c}20 \mathrm{~nm} \text { ALD on } \\
30 \mathrm{~nm} \text { PVD }\end{array}$ & $6.4 \pm 0.4$ & $19 \pm 1$ & $\begin{array}{c}0.37 \pm 8 \mathrm{E}- \\
3\end{array}$ & $1.39 \pm 0.09$ \\
\hline PVD & $50 \mathrm{~nm}$ PVD & $12.4 \pm 0.8$ & $112 \pm 10$ & $\begin{array}{c}1.36 \pm 0.1 \\
6\end{array}$ & $1.37 \pm 0.10$ \\
\hline
\end{tabular}

\section{Table 4}

\begin{tabular}{|c|c|c|c|}
\hline Sample ID & $\begin{array}{c}\text { Film Thickness } \\
(\mu \mathrm{m})\end{array}$ & $\boldsymbol{R}_{\boldsymbol{T} \boldsymbol{x}}$ & $\boldsymbol{\tau}_{\mathbf{5 0}}$ (hours) \\
\hline ALD-20 & $0.33 \pm 0.06$ & $4.0 \pm 0.2$ & $48 \pm 5$ \\
\hline ALD-40 & $0.64 \pm 0.05$ & $5.1 \pm 0.2$ & $12.0 \pm 1.2$ \\
\hline ALD-60 & $0.85 \pm 0.08$ & $5.9 \pm 0.1$ & $8.2 \pm 0.8$ \\
\hline ALD-80 & $1.00 \pm 0.11$ & $6.4 \pm 0.1$ & $7.4 \pm 0.5$ \\
\hline
\end{tabular}

Table 5

\begin{tabular}{|c|c|c|c|c|c|c|}
\hline Sample ID & $\begin{array}{c}\text { Diffusion } \\
\text { Barrier }\end{array}$ & $\begin{array}{c}\text { Seed Layer } \\
\text { Stack }\end{array}$ & $\tau_{50}$ (hours) & $\boldsymbol{R}_{\boldsymbol{T} x}$ & $\begin{array}{c}\overline{\mathrm{I}}_{111} \text { of } \\
\text { Seed Layer } \\
\text { (ct) }\end{array}$ & $\begin{array}{c}\text { Plated Film } \\
\text { Thickness }(\mu \mathrm{m})\end{array}$ \\
\hline PVD-Co & standard & $\begin{array}{c}20 \mathrm{~nm} \text { Evap Co on } \\
30 \mathrm{~nm} \text { PVD Cu }\end{array}$ & $8.4 \pm 0.9$ & $31 \pm 2$ & $0.35 \pm .01$ & $1.49 \pm 0.16$ \\
\hline $\begin{array}{c}\text { PVD- } \\
\text { Evap-Cu }\end{array}$ & standard & $\begin{array}{c}20 \mathrm{~nm} \text { Evap Cu on } \\
30 \mathrm{~nm} \text { PVD Cu }\end{array}$ & $7.6 \pm 0.7$ & $25 \pm 4$ & $0.43 \pm .01$ & $1.73 \pm 0.29$ \\
\hline PVD- $\beta-T a$ & $10 \mathrm{~nm} \beta-T a$ & $50 \mathrm{~nm}$ PVD & $9.1 \pm 0.2$ & $55 \pm 4$ & $4.0 \pm 0.25$ & $1.34 \pm 0.16$ \\
\hline
\end{tabular}

Table 6

\begin{tabular}{|c|c|c|c|}
\hline Sample ID & Film Thickness & $\tau_{\text {50 }}$ (hours) & $\boldsymbol{R}_{\boldsymbol{T x}}$ \\
\hline I-PVD-400 & $0.55 \pm 0.03 \mu \mathrm{m}$ & $25 \pm 1$ & $335 \pm 19$ \\
\hline I-PVD-600 & $0.85 \pm 0.05 \mu \mathrm{m}$ & $4.4 \pm 0.1$ & $37.6 \pm 9.3$ \\
\hline I-PVD-800 & $1.14 \pm 0.07 \mu \mathrm{m}$ & $5.5 \pm 0.2$ & $33.4 \pm 7.9$ \\
\hline I-PVD-1000 & $1.48 \pm 0.02 \mu \mathrm{m}$ & $4.0 \pm 0.1$ & $14.7 \pm 0.3$ \\
\hline
\end{tabular}

Table 7 


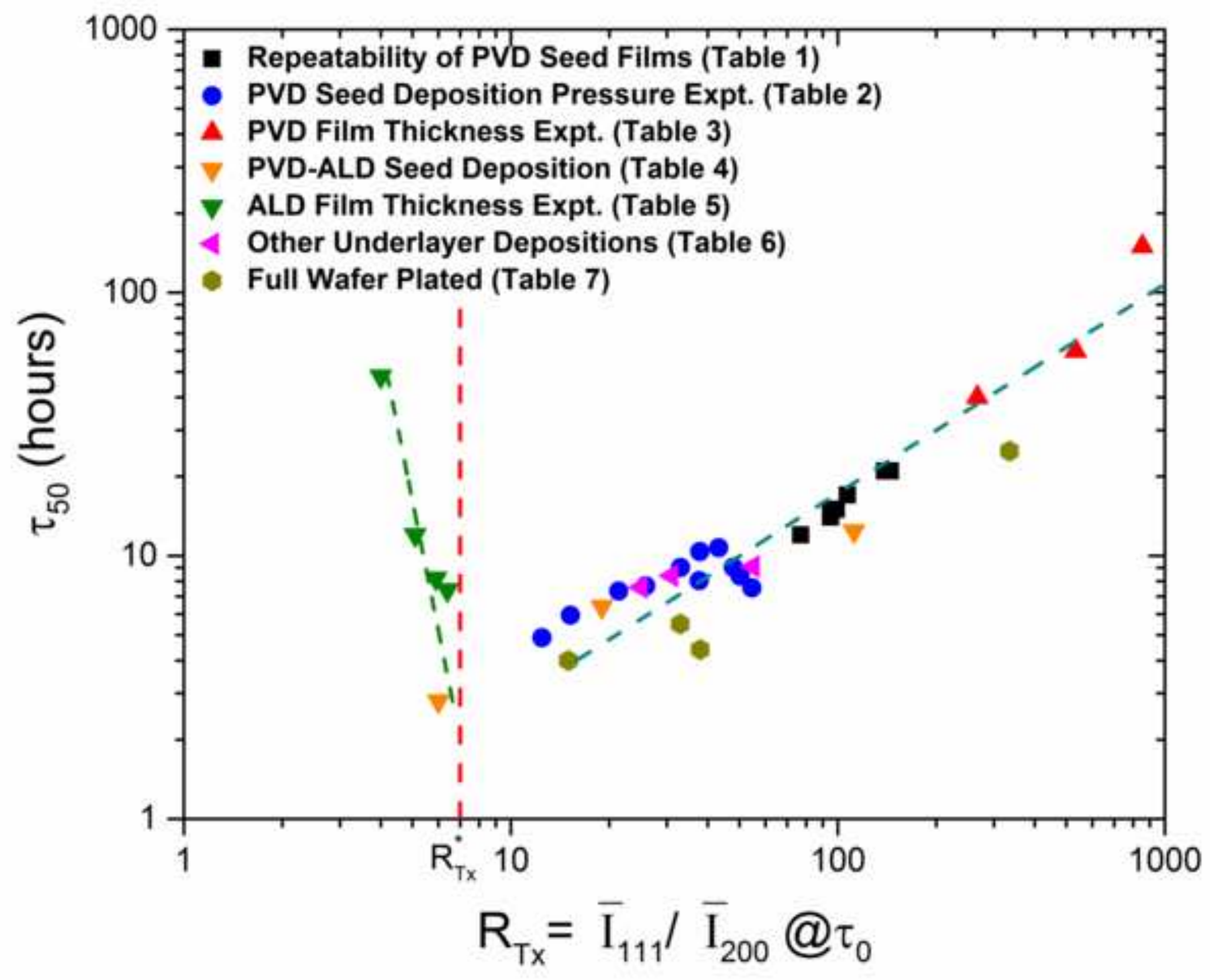




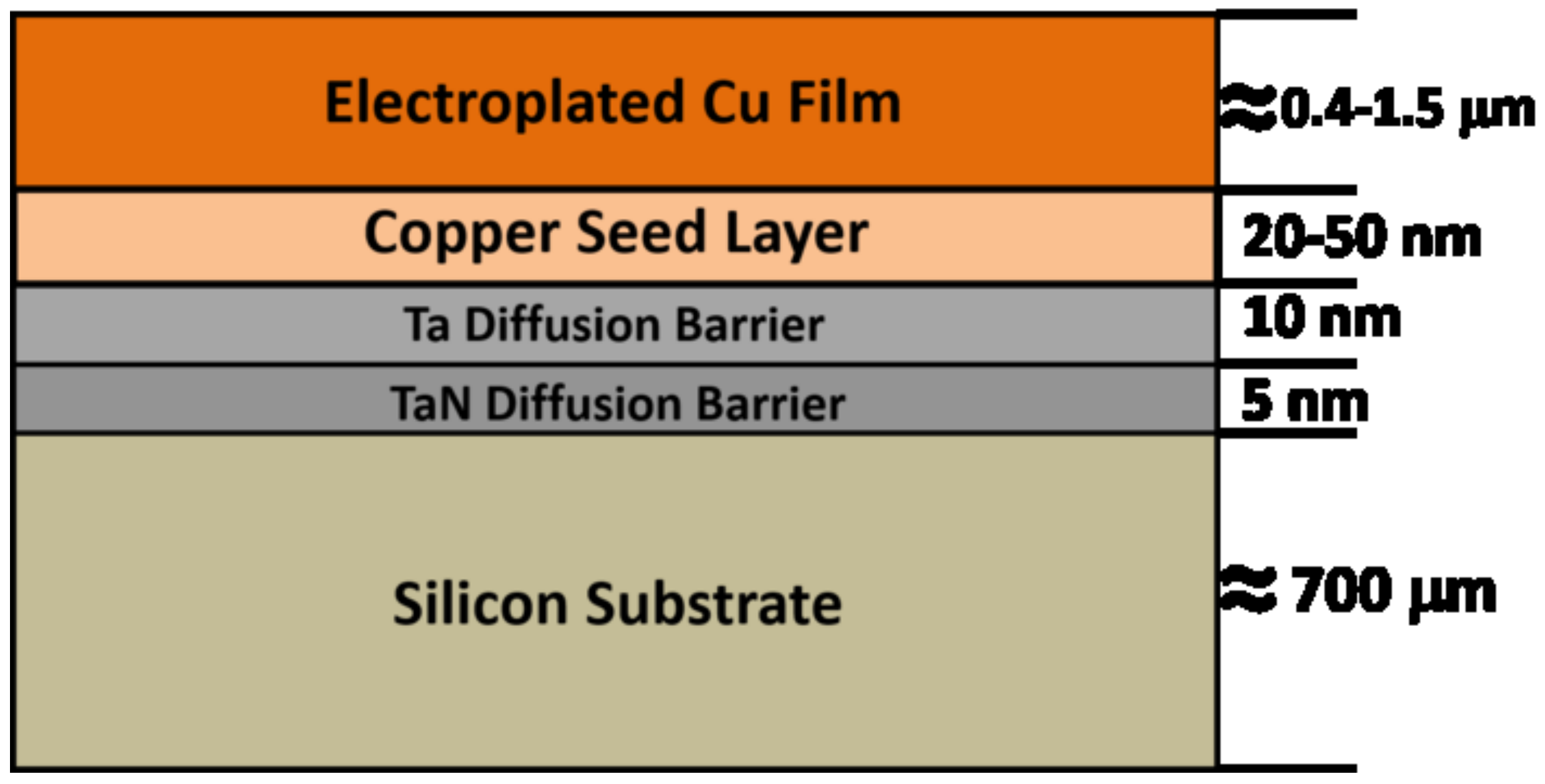

Electroplated Cu Film

20-50 $\mathrm{nm}$

$10 \mathrm{~nm}$

$5 \mathrm{~nm}$

Silicon Substrate

$700 \mu \mathrm{m}$

Copper Seed Layer

Ta Diffusion Barrier 


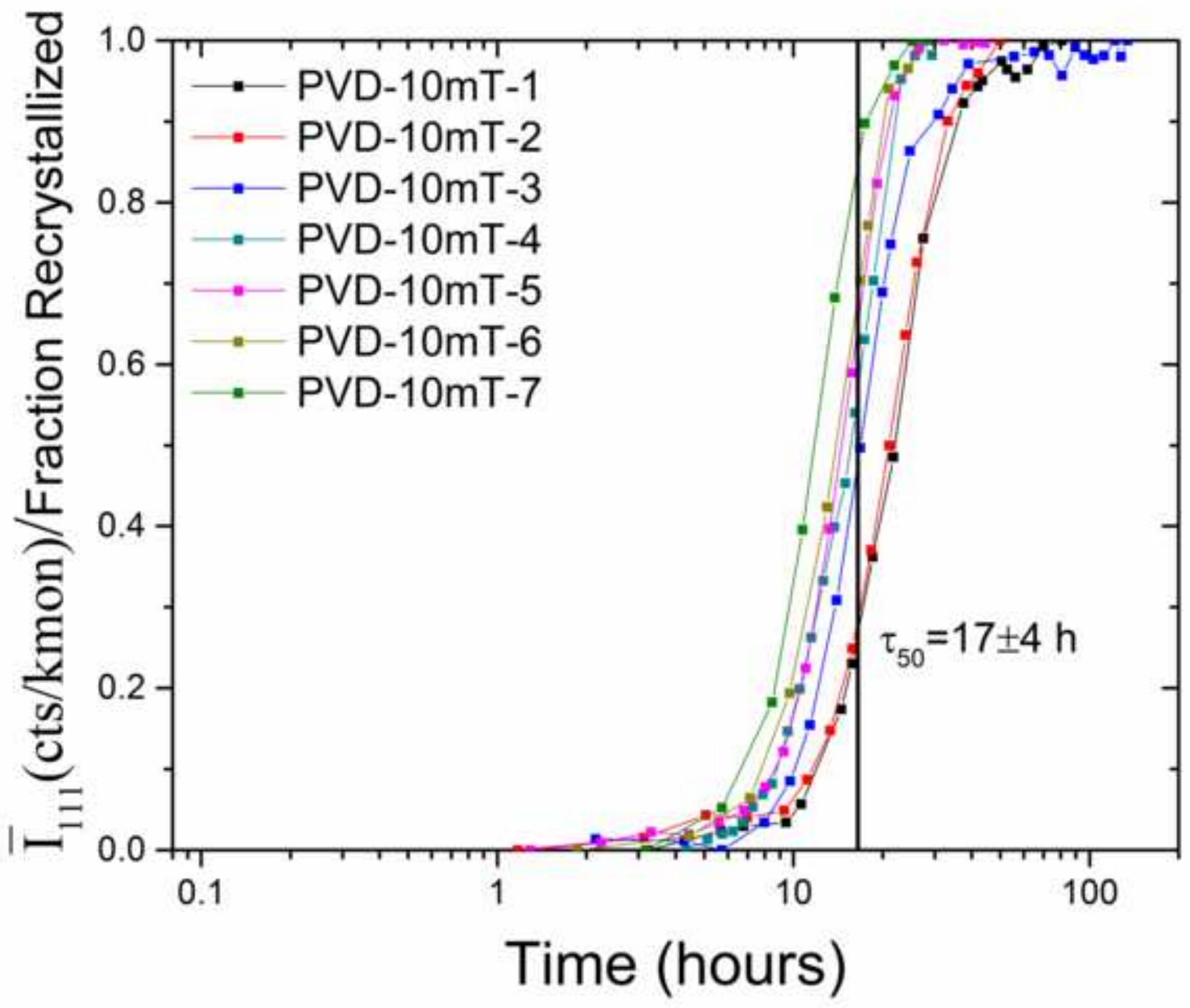




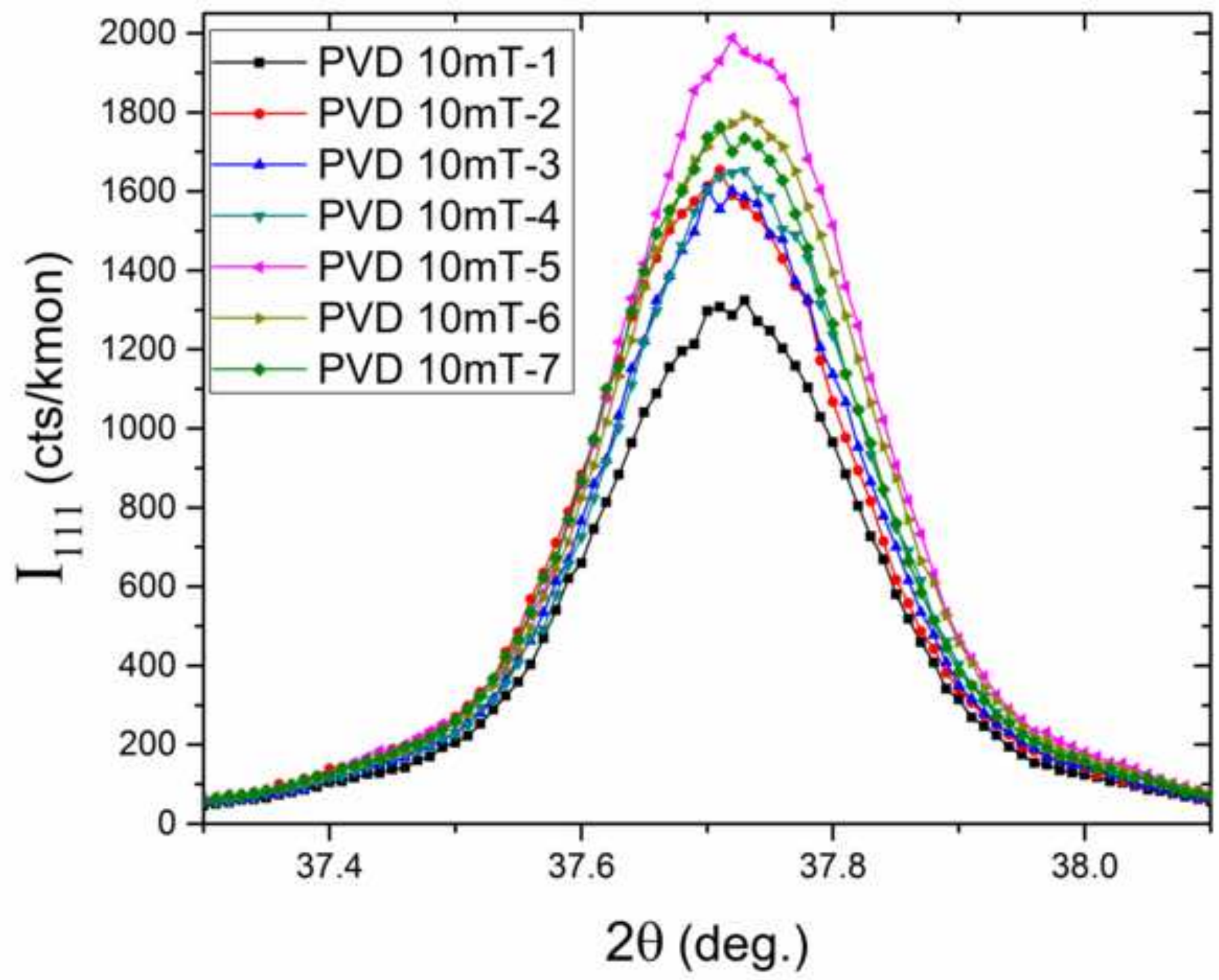




\section{$H_{2}=20.10 \mathrm{~nm}$}

CUSEEDK40 / 2REA

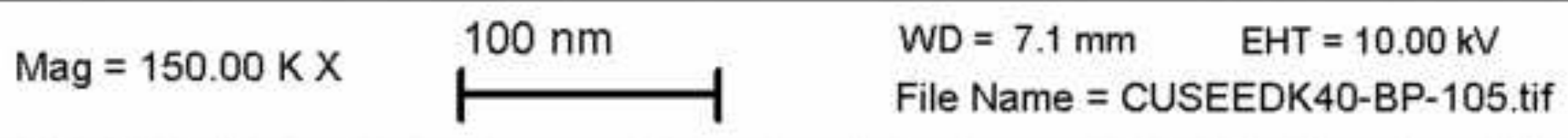




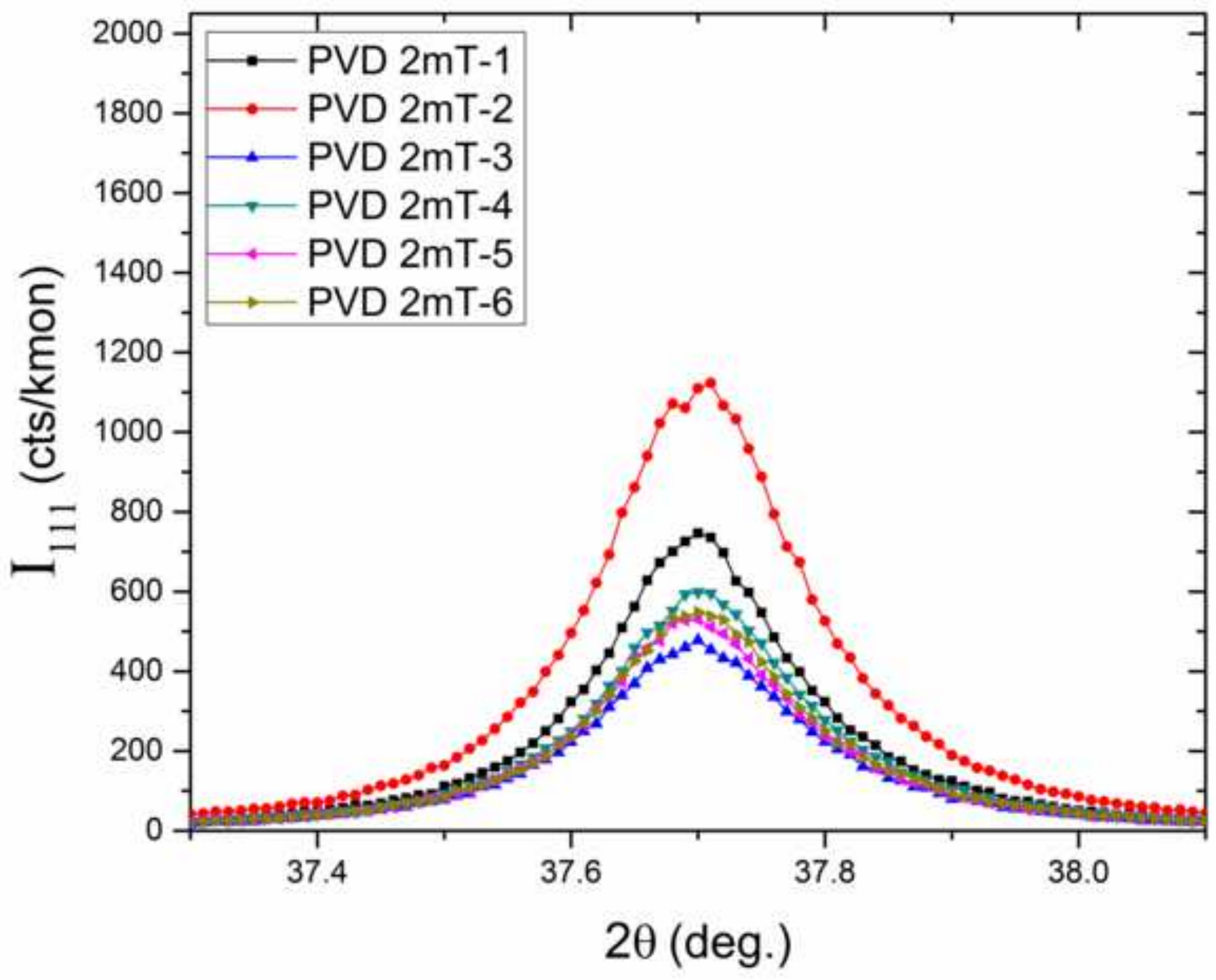




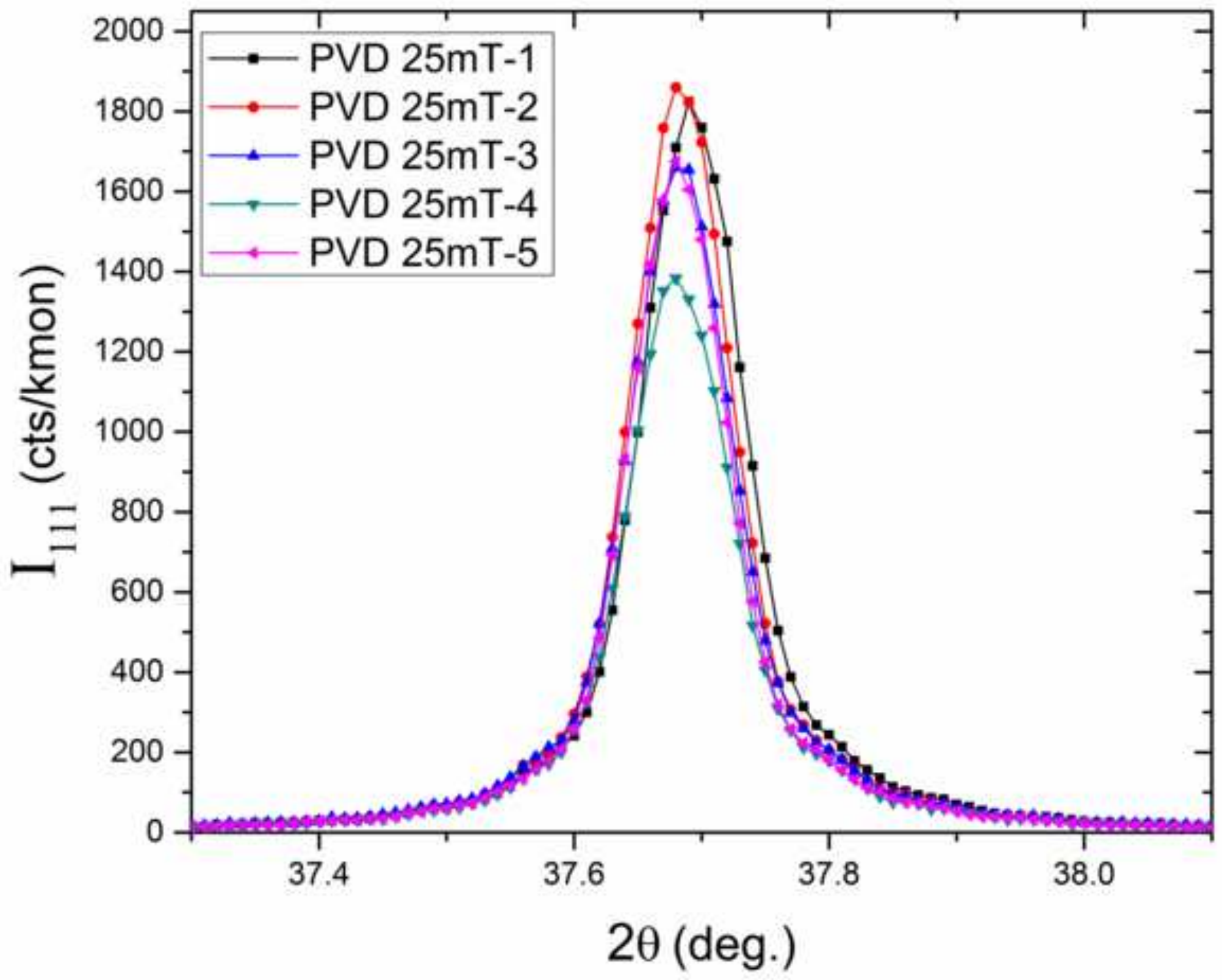




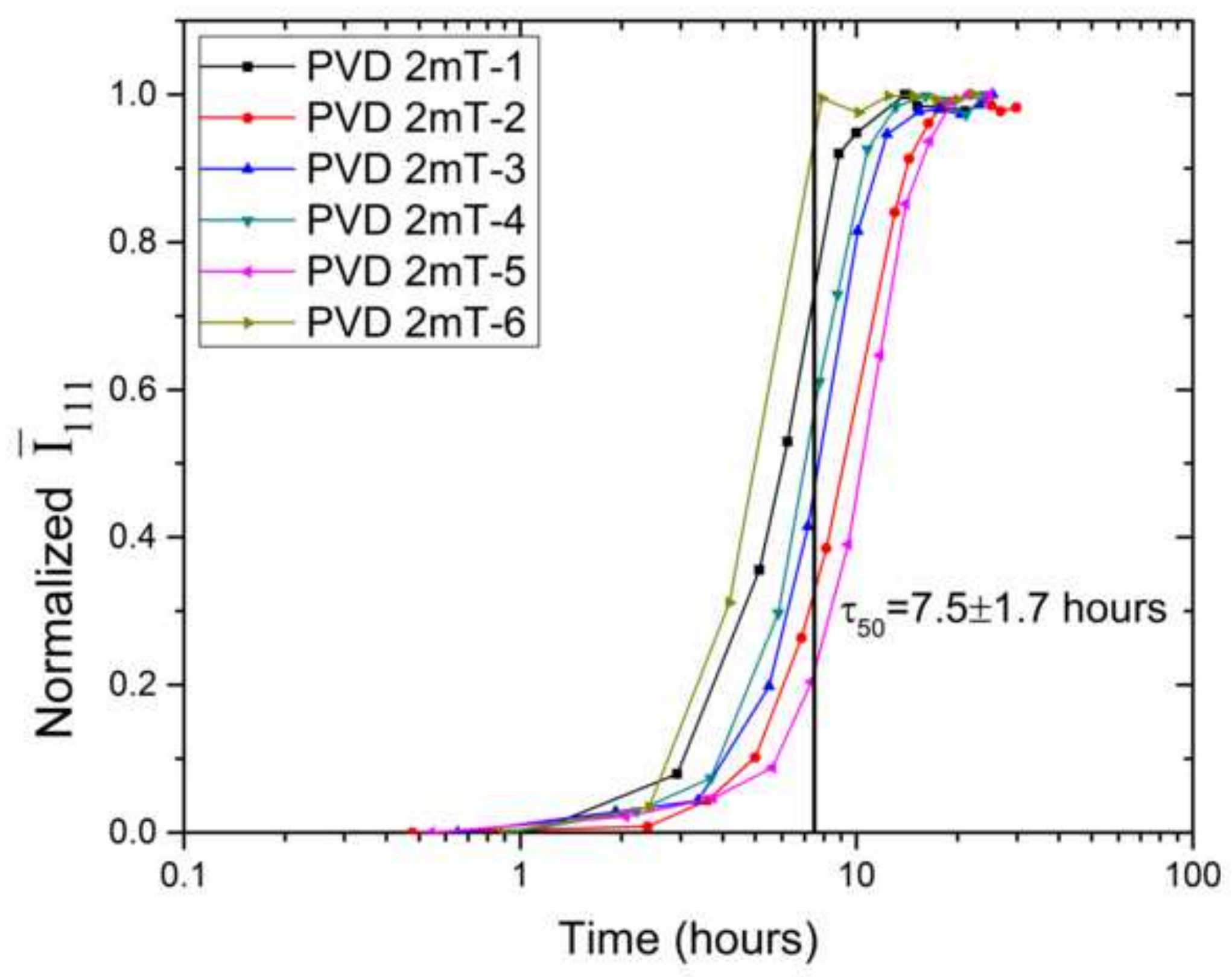




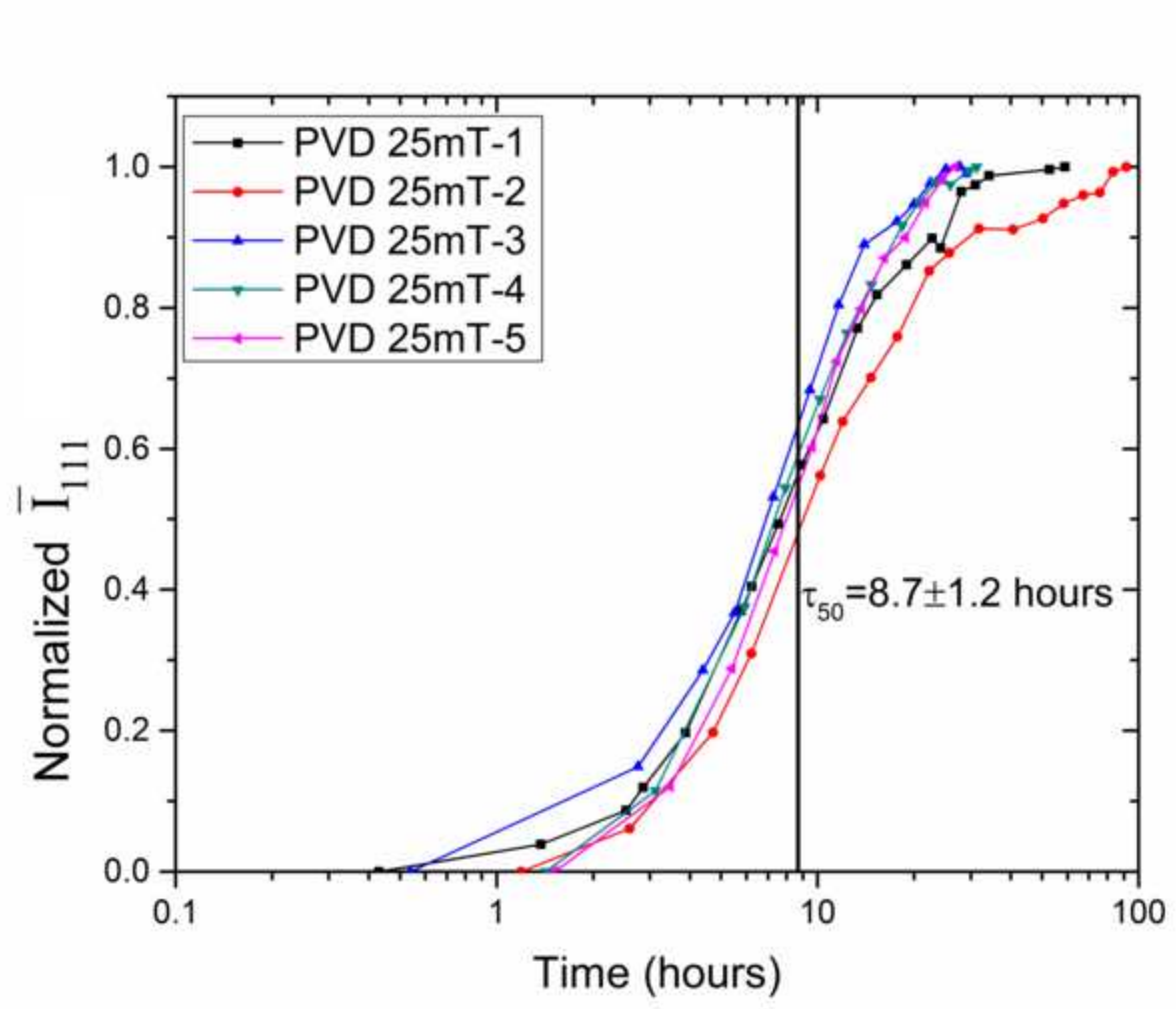




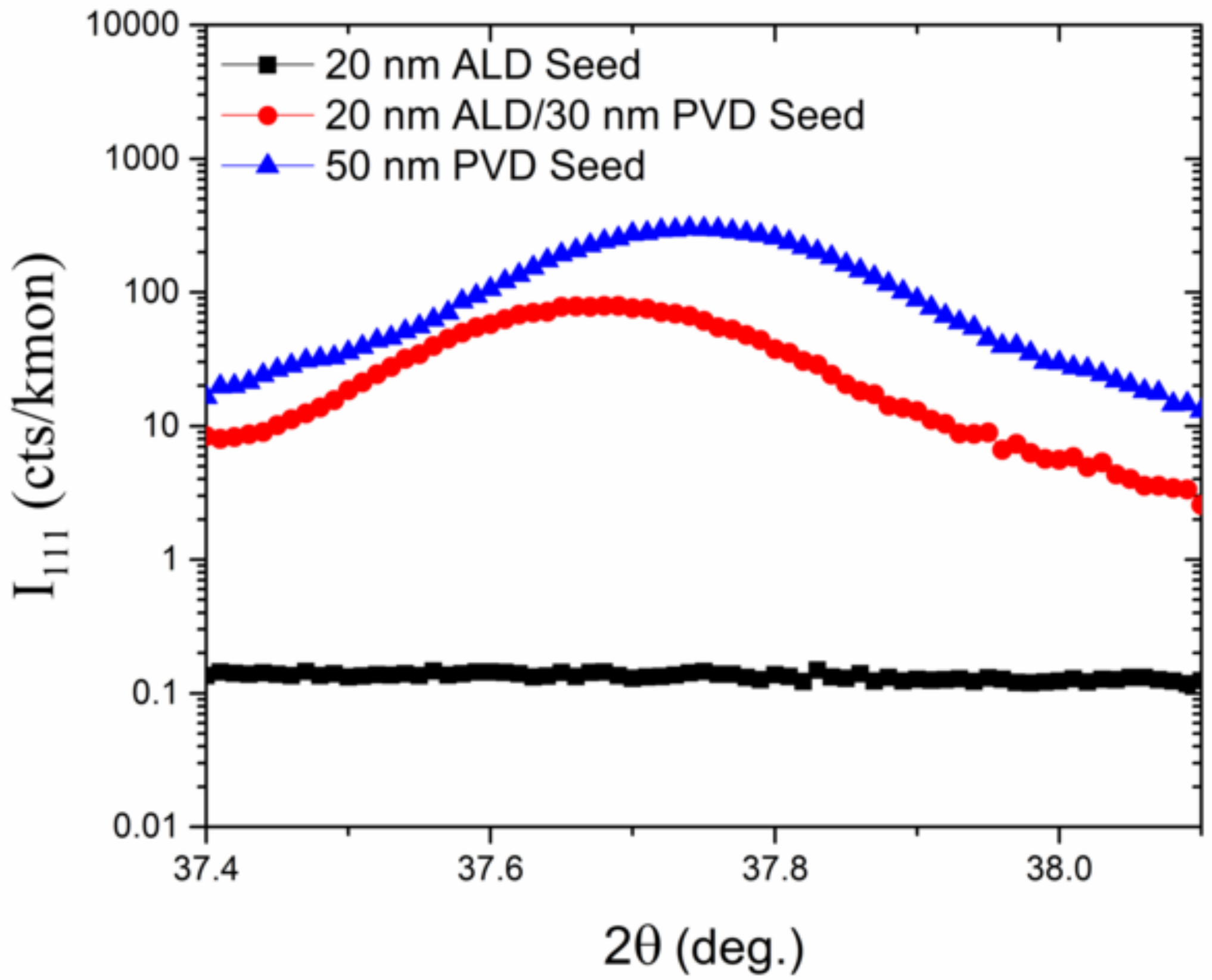




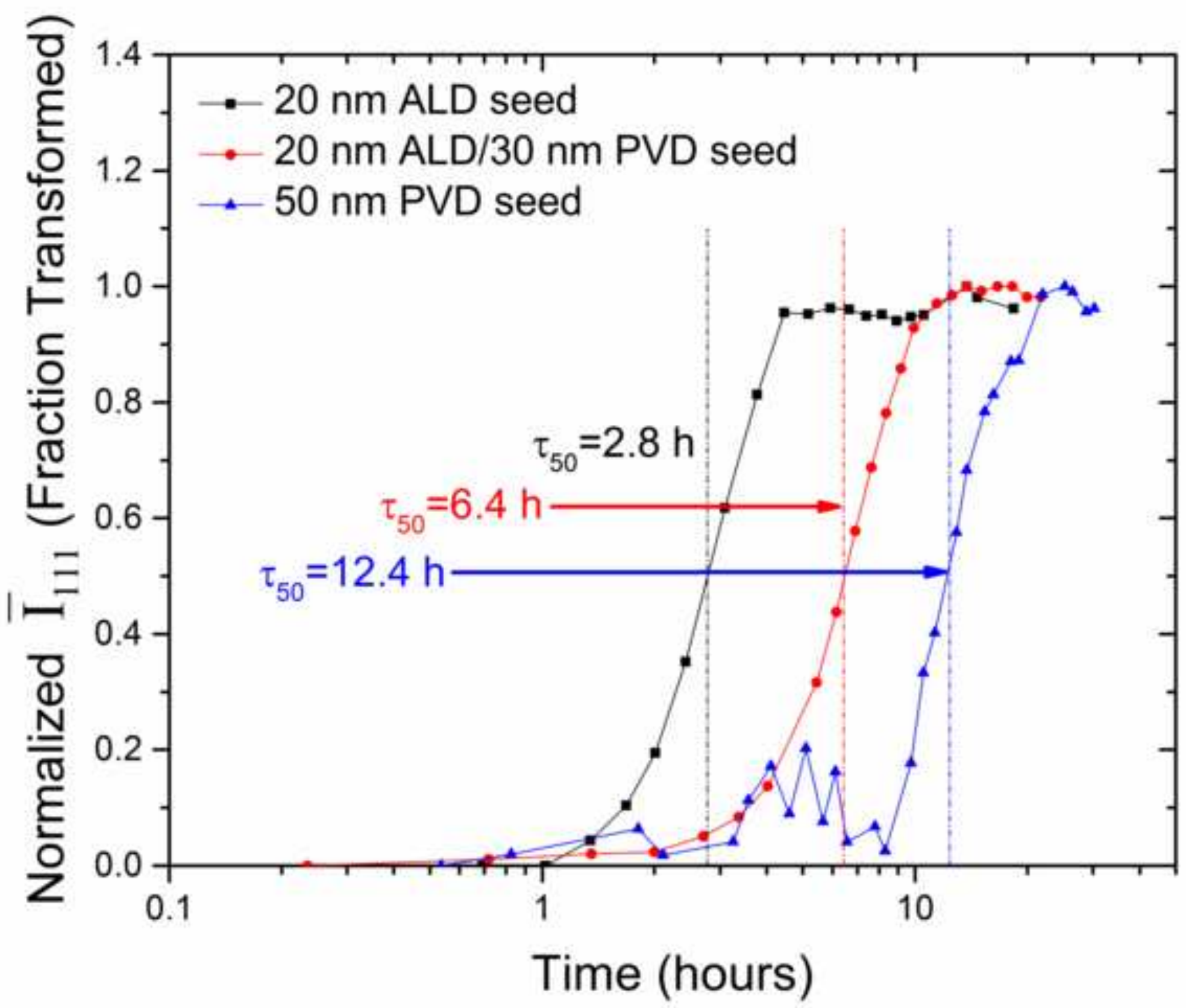




\section{BNLSEMK42 / V33A}

WD $=5.2 \mathrm{~mm} \quad E H T=10.00 \mathrm{KV}$

File Name $=$ BNLSEMK42-BP-107.

$$
\text { Mag }=200.00 \mathrm{KX}
$$

\section{Figure}

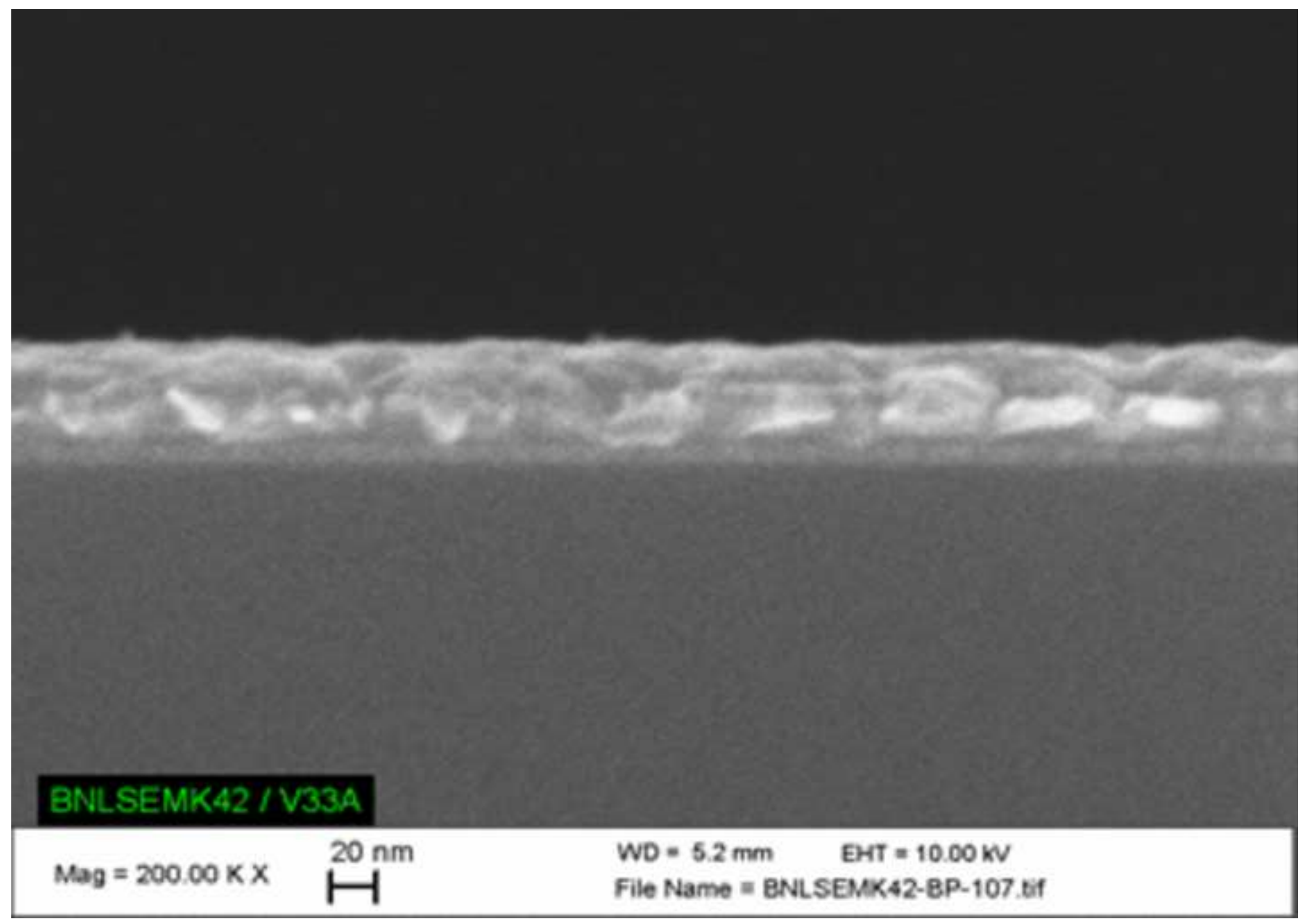




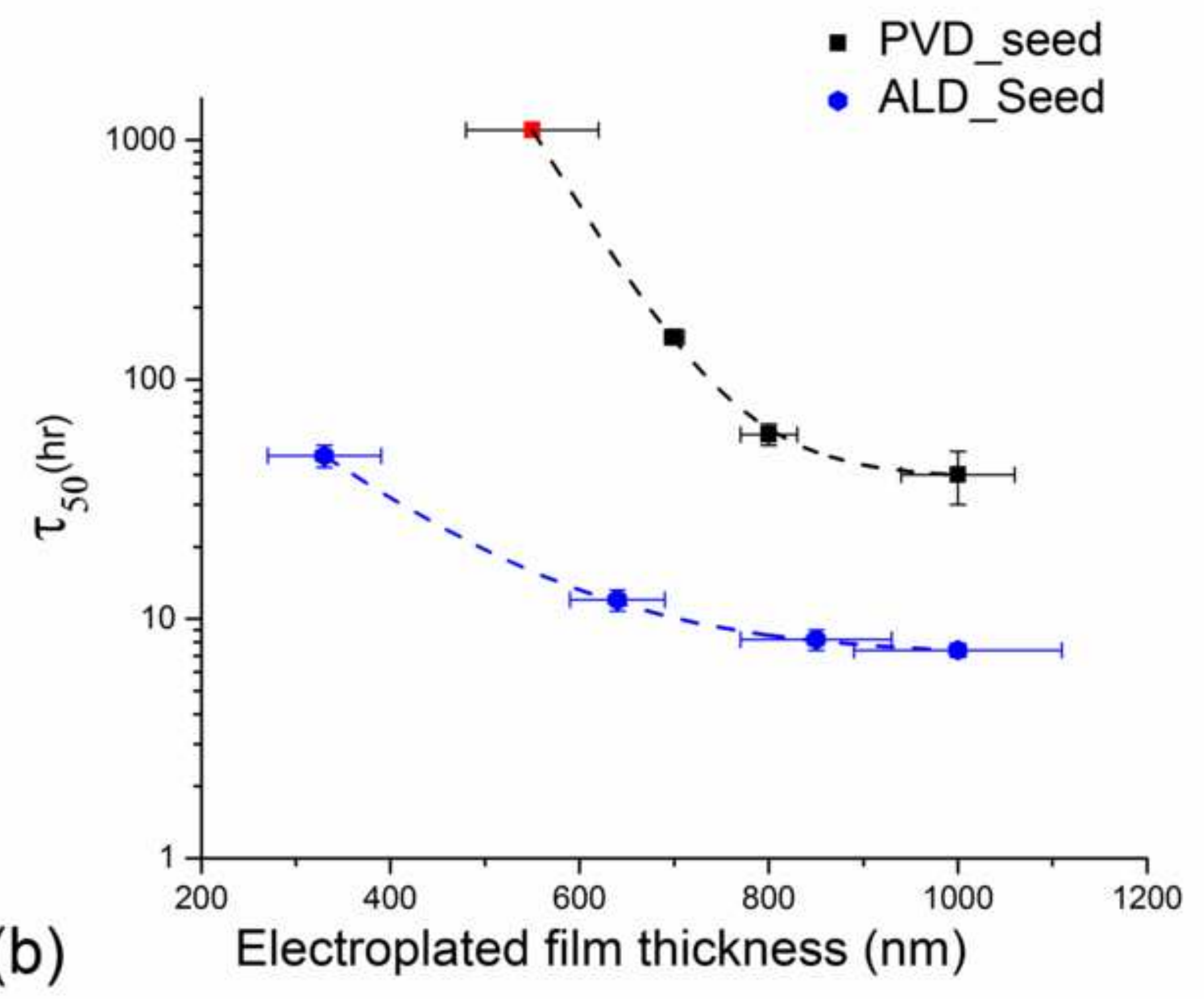

(b) Electroplated film thickness $(\mathrm{nm})$

- PVD_seed

- ALD_Seed C

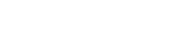

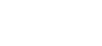
(n) 